\title{
Uniform Semiclassical Approximation for the Wigner $6 j$ Symbol in Terms of Rotation Matrices
}

\author{
Robert G. Littlejohn \\ Liang $\mathrm{Yu}$ \\ University of California \\ Berkeley, CA 94720-7300 USA
}

November 9, 2018

\begin{abstract}
A new uniform asymptotic approximation for the Wigner $6 j$ symbol is given in terms of Wigner rotation matrices ( $d$-matrices). The approximation is uniform in the sense that it applies for all values of the quantum numbers, even those near caustics. The derivation of the new approximation is not given, but the geometrical ideas supporting it are discussed and numerical tests are presented, including comparisons with the exact $6 j$-symbol and with the Ponzano-Regge approximation.
\end{abstract}

\section{Introduction}

The Wigner $6 j$-symbol is used in the recoupling of three angular momenta, and finds many applications in atomic, molecular and nuclear physics. These are explained in standard references on angular momentum theory 1, 2, 3, 4. For example, the $3 n j$-symbols and their asymptotic properties are central to certain algorithms for the calculation of scattering amplitudes in three-body interactions (De Fazio et al[5], Anderson and Aquilanti[6]). These methods make use of the relationship between the $3 n j$-symbols and discrete orthogonal polynomials (Aquilanti et al[7, 8, 9] and references therein). 
The $6 j$-symbol possesses a remarkable semiclassical approximation, first obtained by Ponzano and Regge[10] through some inspired guesswork, that is linked in a highly symmetrical manner to the geometry of a tetrahedron in three-dimensional space. This formula was first proven rigorously by Schulten and Gordon[11], who also gave practical means of computing the $6 j$-symbol by recursion relations. More recently the $6 j$-symbol has attracted attention for the role it plays in quantum gravity, which has led to more geometrical treatments of its asymptotic properties. References in this area include Roberts[12] and Charles[13].

The formula of Ponzano and Regge has the usual properties of primitive semiclassical approximations, for example, it diverges at the classical turning points (the caustics). Since the $6 j$-symbol is only defined for discrete values of the quantum numbers, it is unlikely to fall exactly on a caustic, but it can come very close, and the Ponzano-Regge formula does not provide a good approximation for such values. Thus there is interest in uniform approximations that do not suffer from caustic singularities and that are valid over as wide a range of quantum numbers as possible.

In addition to proving the Ponzano-Regge formula for the $6 j$-symbol, Schulten and Gordon also provided uniform asymptotic approximations of the Airy-function type that are valid in a region passing through a turning point. The $6 j$-symbol, however, has two turning points when one of the $j$ 's is varied and the others held fixed, in a manner reminiscent of an ordinary oscillator in one dimension. The Airy-function uniform approximation can only cover one of these at a time, and if two Airy-function approximations are used, then they do not match smoothly in the middle. This suggests that a uniform approximation of the Weber-function type (one based on harmonic oscillator wave functions) should be used, that would cover both turning points at once.

It turns out, however, that this cannot be done, in general. An idea of why this is so is given by Fig. 1, which shows the $6 j$-symbol and the PonzanoRegge approximation as a function of $j_{12}$ for certain values of the other five $j$ 's (notation is explained by (11) below). The sticks in the diagram give the exact values of the $6 j$-symbol, while the curve is the Ponzano-Regge approximation. Although the $6 j$-symbol is only defined for discrete values of $j_{12}$, the PonzanoRegge approximation is defined for continuous values of the parameters, and allows us to talk of the phase of the $6 j$-symbol in an unambiguous manner. The Ponzano-Regge approximation shows the expected divergences at the caustics or turning points, and the Airy function behavior at the right turning 
point. In the classically allowed region between the turning points the wave length decreases as we move to the left, corresponding to an increase in the variable conjugate to $j_{12}$ (the angle $\phi_{12}$, defined in Sec. 2.4below). At the left turning point the Airy function is not so obvious, because it is multiplied by a rapidly oscillating cosine term. This is because the angle $\phi_{12}$ conjugate to $j_{12}$ reaches the value $\pm \pi$ at the right turning point.

The behavior illustrated in Fig. 1 1 cannot be matched by a Weber function (harmonic oscillator eigenfunction), for which both turning points have the behavior of an unmodulated Airy function. Related to this is the fact that the difference between the Ponzano-Regge phases at the two turning points is not of the form $\left(n+\frac{1}{2}\right) \pi$ for the parameters shown in Fig. 1, For harmonic oscillator eigenfunctions the difference in the action between the two turning points is always of the form $\left(n+\frac{1}{2}\right) \pi$, a condition that is equivalent to the single-valuedness of the WKB wave function (that is, it is the BohrSommerfeld quantization condition). One can say that the $6 j$-symbol has a nonstandard matching condition of the two WKB branches at the lower turning point for the parameters in Fig. 1,

Ultimately there are topological reasons for the failure of the Weber function as a standard form for a uniform approximation in cases such as that illustrated in Fig. 1. Uniform approximations are based on a smooth, area preserving map between the phase space of the given problem and the standard problem. But the phase space of the $6 j$-symbol is a sphere (we call it the $6 j$-sphere), and the phase space of the harmonic oscillator is a plane. These two spaces cannot be continuously mapped into one another.

We have noticed, however, that the phase space that arises in the semiclassical analysis of the Wigner $d$-matrices (rotation matrices) is also a sphere. We call this the $d$-sphere, to distinguish it from the $6 j$-sphere. Moreover, the classical orbits on the two spheres are topological circles in both cases, and in both cases intersecting orbits always intersect in two points, unless they are tangent, in which case there is one intersection point. There are also cases in which the orbits do not intersect at all, corresponding to classically forbidden regions. As is well known, the classical orbits and their intersections provide the geometrical framework for the construction of semiclassical (asymptotic) approximations. These topological features are the same for both the $6 j$ sphere and the $d$-sphere, suggesting that one can be mapped into the other by a smooth transformation that takes a pair of orbits on one sphere into the pair of orbits on the other.

We have worked out the details of this mapping and the corresponding 
uniform approximation. The resulting approximation is smooth and uniform over the entire range of quantum numbers $j_{12}$ and $j_{23}$ (for fixed $j_{1}, j_{2}, j_{3}$ and $j_{4}$ ). For most values of the $j$ 's it is more accurate than the Ponzano-Regge approximation, certainly near the caustics but also at most other places. We have found no places where it is dramatically worse than the Ponzano-Regge approximation.

In this paper we shall present the new uniform approximation itself, as well as some of the geometric rationale behind it, which helps considerably in understanding the formula and the various regions that it covers. In addition, we shall present some numerical tests of the new formula and comparisons with the exact $6 j$-symbol and the Ponzano-Regge approximation. We shall not, however, present the details of the derivation.

The outline of this paper is as follows. In Sec. 2 we present a collection of facts about the $6 j$-symbol and its asymptotic or semiclassical approximation (the Ponzano-Regge formula), emphasizing the spherical phase space and its geometrical ramifications. In Sec. 3 we present a geometrical treatment of the asymptotic properties of the rotation matrices ( $d$-matrices) that emphasizes the similarities with the $6 j$-symbol. In Sec. 4 we outline the ideas behind the uniform approximation of the $6 j$-symbol in terms of $d$-matrices, we present the actual formulas, and we present some numerical tests. Finally, in Sec. 5 we make some conclusions.

\section{The $6 j$-symbol}

\subsection{Quantum Mechanics of the $6 j$-symbol}

We set $\hbar=1$, so all angular momenta are dimensionless. We label the $j$ 's in the $6 j$ symbol by

$$
\left\{\begin{array}{lll}
j_{1} & j_{2} & j_{12} \\
j_{3} & j_{4} & j_{23}
\end{array}\right\},
$$

which is how it would used when recoupling three angular momenta.

The quantum number $j_{i}, i=1,2,3,4,12,23$, just gives the magnitude of the angular momentum, and does not specify the sign of the operator. For example, instead of coupling three angular momenta to obtain a fourth, that is, setting $\mathbf{J}_{4}=\mathbf{J}_{1}+\mathbf{J}_{2}+\mathbf{J}_{3}$, we can couple four angular momenta with a sum of zero,

$$
\mathbf{J}_{1}+\mathbf{J}_{2}+\mathbf{J}_{3}+\mathbf{J}_{4}=0
$$


(effectively changing the sign of $\mathbf{J}_{4}$ ). This is how we shall regard the recoupling problem in this paper. Usually we will think of $j_{i}, i=1,2,3,4$ as given, while $j_{12}$ and $j_{23}$ are variable intermediate angular momenta that result from the coupling of the first four. They are the quantum numbers of the squares of the operators

$$
\mathbf{J}_{12}=\mathbf{J}_{1}+\mathbf{J}_{2}, \quad \mathbf{J}_{23}=\mathbf{J}_{2}+\mathbf{J}_{3} .
$$

With this interpretation, the $6 j$-symbol in the form (1) is proportional to the unitary matrix element $\left\langle j_{12} \mid j_{23}\right\rangle$ that takes one from the eigenbasis of one intermediate angular momentum $\left(j_{12}\right)$ to the eigenbasis of the other $\left(j_{23}\right)$. These bases span the subspace of the product space of four angular momenta in which (2) holds as an operator equation. We shall denote this subspace by $Z$. According to (2), the total angular momentum vanishes on $Z$. The orthonormality relations satisfied by the $6 j$-symbol (see, for example, Edmonds [1] Eq. (6.2.9)) are essentially a statement of the unitarity of the matrix $\left\langle j_{12} \mid j_{23}\right\rangle$.

To be defined the $6 j$-symbol (1) must satisfy four triangle inequalities, in $\left(j_{1}, j_{2}, j_{12}\right),\left(j_{2}, j_{3}, j_{23}\right),\left(j_{3}, j_{4}, j_{12}\right)$, and $\left(j_{1}, j_{4}, j_{23}\right)$, for example, $j_{12}$ must lie between the bounds

$$
\left|j_{1}-j_{2}\right| \leq j_{12} \leq j_{1}+j_{2}
$$

in integer steps. For given $j_{i}, i=1,2,3,4$, these imply that $j_{12}$ and $j_{23}$ vary between the limits

$$
\begin{aligned}
& j_{12, \text { min }} \leq j_{12} \leq j_{12, \max } \\
& j_{23, \text { min }} \leq j_{23} \leq j_{23, \max }
\end{aligned}
$$

in integer steps, where

$$
\begin{aligned}
j_{12, \min } & =\max \left(\left|j_{1}-j_{2}\right|,\left|j_{3}-j_{4}\right|\right), \\
j_{23, \min } & =\max \left(\left|j_{2}-j_{3}\right|,\left|j_{1}-j_{4}\right|\right), \\
j_{12, \max } & =\min \left(j_{1}+j_{2}, j_{3}+j_{4}\right), \\
j_{23, \max } & =\min \left(j_{2}+j_{3}, j_{1}+j_{4}\right) .
\end{aligned}
$$

The number of allowed $j_{12}$ or $j_{23}$ values is the same, and it is the dimension $D$ of the subspace $Z$ as well as the size of the matrix $\left\langle j_{12} \mid j_{23}\right\rangle$,

$$
D=\operatorname{dim} Z=j_{12, \max }-j_{12, \min }+1=j_{23, \max }-j_{23, \min }+1 \text {. }
$$




\subsection{Classical and Semiclassical Mechanics of the $6 j$ - symbol}

The basic reference on the semiclassical mechanics of the $6 j$-symbol is Ponzano and Regge[10]. We add to their discussion an appreciation of the Gram matrix (see Appendix $\mathrm{A}$ ) and the recent realization that the phase space of the $6 j$-symbol is a sphere [13].

We shall reserve lower case $j_{i}$ for quantum numbers as in (1), and for semiclassical purposes we shall set

$$
J_{i}=j_{i}+\frac{1}{2}
$$

(with capital $J$ 's), for $i=1,2,3,4,12,23$. The quantity $J_{i}$ is interpreted as the length of the classical angular momentum vector associated with the quantum number $j_{i}$. The correction $1 / 2$ is a Maslov index, and the manner in which it arises in the semiclassical theory of angular momentum is explained in Aquilanti et al[14.

We shall write $\mathbf{J}_{i}$ (in bold face) either for the vector of angular momentum operators in quantum mechanics, or for the classical angular momentum vector in a classical model. The distinction will be established by context. For example, in the classical context, (2) is interpreted as a constraint on the four classical vectors $\mathbf{J}_{i}, i=1,2,3,4$, while (3) is interpreted as the definitions of two more classical angular momenta $\mathbf{J}_{12}$ and $\mathbf{J}_{23}$.

The condition (8) is a quantization condition of the Bohr-Sommerfeld type, restricting the the classical quantity $J_{i}$ to discrete values. Some authors have viewed (8) as an approximation to $[j(j+1)]^{1 / 2}$, valid when $j$ is large, but in fact it is represents the exact eigenvalues of a certain operator for all values of $j$, even $j=0$. When properly understood [14], (8) is equivalent to the fact that the eigenvalues of the operator $J^{2}$ are $j(j+1)$.

In purely classical mechanics, however, there is no quantization, and all variables take on continuous values. We must allow this in order to view the classical phase space. To visualize the phase space of the $6 j$-symbol, we will assume that $J_{i}, i=1,2,3,4$ have any fixed positive values, while $J_{12}$ and $J_{23}$ are variables, the lengths of the vectors $\mathbf{J}_{12}$ and $\mathbf{J}_{23}$ defined by (3). Then $J_{12}$ and $J_{23}$ are restricted by classical versions of the triangle inequalities,

$$
\begin{aligned}
& J_{12, \text { min }} \leq J_{12} \leq J_{12, \max } \\
& J_{23, \min } \leq J_{23} \leq J_{23, \max }
\end{aligned}
$$


where $J_{12}$ and $J_{23}$ vary continuously between the bounds indicated, and where the bounds themselves are given by

$$
\begin{aligned}
J_{12, \text { min }} & =\max \left(\left|J_{1}-J_{2}\right|,\left|J_{3}-J_{4}\right|\right), \\
J_{23, \text { min }} & =\max \left(\left|J_{2}-J_{3}\right|,\left|J_{1}-J_{4}\right|\right), \\
J_{12, \max } & =\min \left(J_{1}+J_{2}, J_{3}+J_{4}\right), \\
J_{23, \max } & =\min \left(J_{2}+J_{3}, J_{1}+J_{4}\right) .
\end{aligned}
$$

These are the bounds of $J_{12}$ and $J_{23}$ on the $6 j$-sphere.

Here are two useful theorems. First, if $J_{23 \text {,min }}=J_{1}-J_{4}$ or $J_{2}-J_{3}$, then $J_{12, \text { max }}=J_{3}+J_{4}$, otherwise $J_{12, \max }=J_{1}+J_{2}$. Second, if $J_{12 \text {,min }}=J_{1}-J_{2}$ or $J_{4}-J_{3}$, then $J_{23, \max }=J_{2}+J_{3}$, otherwise $J_{23, \max }=J_{1}+J_{4}$.

If the quantization conditions (8) hold for $J_{i}, i=1,2,3,4$, then the bounds on the continuous variables $J_{12}$ and $J_{23}$ can be expressed in terms of the bounds on the quantum number $j_{12}$ and $j_{23}$ by

$$
\begin{array}{ll}
J_{12, \text { min }}=j_{12, \text { min }}, & J_{12, \text { max }}=j_{12, \text { max }}+1, \\
J_{23, \text { min }}=j_{23, \text { min }}, & J_{23, \text { max }}=j_{23, \text { max }}+1 .
\end{array}
$$

Combined with (7), these imply

$$
D=J_{12, \max }-J_{12, \min }=J_{23, \max }-J_{23, \text { min }} .
$$

If $J_{i}>0, i=1,2,3,4$ and if $J_{12}$ and $J_{23}$ satisfy the triangle inequalities (9), then it is possible to find six classical vectors $\mathbf{J}_{i}, i=1,2,3,4,12,23$ that can be placed end-to-end in subsets of three to create four triangles. The triangles are defined by (3), plus $\mathbf{J}_{12}+\mathbf{J}_{3}+\mathbf{J}_{4}=0$ and $\mathbf{J}_{1}+\mathbf{J}_{23}+\mathbf{J}_{4}=0$. In particular, this can always be done when the six $J_{i}$ satisfy the quantization conditions (86) for values of $j_{i}$ that are valid in a $6 j$-symbol, in which case $J_{i}>0$ for all six $i$ (because $j_{i} \geq 0$ ) and the areas of the triangles are positive (because the $j_{i}$ satisfy the triangle inequalities).

For some values of the six $J_{i}$ the four triangles can be fitted together to form the four faces of a tetrahedron. This is the classically allowed region of the $6 j$-symbol. If they can, then the signed volume of the tetrahedron is given by

$$
V=\frac{1}{6} \mathbf{J}_{1} \cdot\left(\mathbf{J}_{2} \times \mathbf{J}_{3}\right)=\frac{1}{6} \mathbf{A}_{1} \cdot\left(\mathbf{A}_{2} \times \mathbf{A}_{3}\right),
$$

where the vectors $\mathbf{A}_{i}$ are defined in $(\underline{86})$. The volume is related to the nonnegative definite Gram matrix $G$ defined in (87) by

$$
36 V^{2}=\operatorname{det} G \text {. }
$$


The tetrahedron is illustrated in Fig. 2, which shows our convention for labeling the edges by classical angular momentum vectors. The vectors in the figure may be seen to satisfy (2) and (3). The tetrahedron in Fig. [2 has positive volume $(V>0)$ according to the definition (13).

For other values of the $J_{i}$ a real tetrahedron does not exist, but a complex tetrahedron exists whose edges are complex vectors $\mathbf{J}_{i}$. These may be chosen so that the $x$ - and $y$-components are purely real and the $z$-component is purely imaginary. This is the classically forbidden region of the $6 j$-symbol. In this case $G$ is still given by (87) and it is still a real, symmetric matrix, but it has one negative eigenvalue. Also, $V^{2}<0$ and $V$ is purely imaginary. Equation (13) is still valid, however, in terms of the complex vectors $\mathbf{J}_{i}$. The dot product of complex vectors $\mathbf{U}$ and $\mathbf{V}$ is defined by $\sum_{i} U_{i} V_{i}\left(\operatorname{not} \sum_{i} U_{i}^{*} V_{i}\right)$, and the length by $J_{i}^{2}=\mathbf{J}_{i} \cdot \mathbf{J}_{i}\left(\right.$ not $\left.\mathbf{J}_{i}^{*} \cdot \mathbf{J}_{i}\right)$.

The classically allowed and forbidden regions are illustrated in Fig. 3 , This figure shows the square region of the $J_{12^{-}} J_{23}$ plane bounded by the classical limits (10) for certain fixed values of $j_{i}, i=1,2,3,4$. The small spots inside the square are the quantized values of $J_{12}$ and $J_{23}$, from which the quantum numbers $j_{12}$ and $j_{23}$ can be extracted by (8). Notice that the quantized values of $J_{12}$ and $J_{23}$ always stay at least one half unit away from the bounds (10). The oval curve is the curve $V=0$, separating the classically allowed from the classically forbidden regions; it is the caustic curve. The classically allowed and forbidden regions lie inside and outside the caustic curve, respectively. Actually, there are four classically forbidden regions, labeled ABCD in the figure. Similar diagrams describe radiative transitions in hydrogen (see Fig. 27 of Ref. [15]).

The caustic curve consists of real tetrahedra that are flat; it touches the square at four points, labeled XYZW in Fig. 3, The geometrical meaning of these points and the behavior of the flat tetrahedron as we move around the caustic curve are illustrated in Fig. 4. At point $Y$, vectors $\mathbf{J}_{1}$ and $\mathbf{J}_{2}$ are antiparallel, giving $\mathbf{J}_{12}$ its minimum length. As $\mathbf{J}_{2}$ rotates in a counterclockwise direction, at first $\mathbf{J}_{12}$ grows and $\mathbf{J}_{23}$ shrinks, as illustrated in the diagram $\mathrm{Y} \rightarrow \mathrm{X}$ in the figure. This is a point between $\mathrm{Y}$ and $\mathrm{X}$ on the caustic line of Fig. 3. When $\mathbf{J}_{2}$ rotates to the angle that causes $\mathbf{J}_{2}$ and $\mathbf{J}_{3}$ to be antiparallel, then $\mathbf{J}_{23}$ is at its minimum length and we are at point X. As $\mathbf{J}_{2}$ continues to rotate, $\mathbf{J}_{23}$ starts to grow again while $\mathbf{J}_{12}$ continues to grow, as illustrated in the diagram $\mathrm{X} \rightarrow \mathrm{Z}$ in the figure. This is a point between $\mathrm{X}$ and $\mathrm{Z}$ on the caustic curve. In this manner we may continue around the caustic curve. 


\subsection{The Ponzano-Regge Formula}

Suppose we are in the classically allowed region so a real tetrahedron exists. Let $\psi_{i}, i=1,2,3,4,12,23$ be the exterior dihedral angles of the tetrahedron associated with edge $\mathbf{J}_{i}$, that is, $\psi_{i}$ is the angle between the outward pointing normals of the two faces that meet in edge $i$, so that each $\psi_{i}$ lies in the interval $[0, \pi]$. Then the Ponzano-Regge phase is defined by

$$
\Phi_{\mathrm{PR}}=\sum_{i} J_{i} \psi_{i}
$$

where the sum runs over all six edges, and the Ponzano-Regge approximation is

$$
\left\{\begin{array}{lll}
j_{1} & j_{2} & j_{12} \\
j_{3} & j_{4} & j_{23}
\end{array}\right\} \approx A_{\mathrm{PR}} \cos \left(\Phi_{\mathrm{PR}}+\frac{\pi}{4}\right),
$$

where the amplitude in the classically allowed region is given by

$$
A_{\mathrm{PR}}=\frac{1}{\sqrt{12 \pi|V|}} .
$$

Given the six quantum numbers $j_{i}$, an algorithm for determining the dihedral angles $\psi_{i}$ is the following. The rules we give are equivalent to those of Ponzano and Regge and Schulten and Gordon, but stated in terms of the diagonalization of the Gram matrix. First we define the six $J_{i}$ by (8) and then we set up the Gram matrix using (88) and diagonalize it. If all the eigenvalues are positive (or if $\operatorname{det} G=36 V^{2}>0$ ), we are in the classically allowed region and we may proceed. Then we construct the six vectors $\mathbf{J}_{i}$ as explained in Appendix A, we compute the outward pointing normals by taking cross products of the vectors spanning the four faces, and finally we compute $\cos \psi_{i}$ as the dot products of the outward pointing normals. This determines $\psi_{i}$ uniquely as an angle in $[0, \pi]$. This is not the most efficient algorithm from a numerical standpoint, since to determine the $\psi_{i}$ only the dot products of the vectors are needed and not the vectors themselves, but it is conceptually clean and has the benefit of allowing one to draw or visualize the tetrahedron itself.

The Ponzano-Regge phase $\Phi_{\mathrm{PR}}$ is continuous inside the classically allowed region, as are the dihedral angles $\psi_{i}$. On the caustic boundary all tetrahedra are flat so all dihedral angles are either 0 or $\pi$. These angles are continuous (hence constant at 0 or $\pi$ ) on the caustic line between points XYZW, but some angles jump discontinuously between 0 and $\pi$ at those points. 


\begin{tabular}{|c||cc|cc|c|cc|}
\hline$i$ & $\mathrm{~A}$ & $\mathrm{~A}$ & $\mathrm{~B}$ & $\mathrm{~B}$ & $\mathrm{C}$ & $\mathrm{D}$ & $\mathrm{D}$ \\
\hline \hline 1 & $\pi$ & 0 & $\pi$ & 0 & $\pi$ & $\pi$ & 0 \\
2 & $\pi$ & 0 & 0 & $\pi$ & $\pi$ & 0 & $\pi$ \\
3 & 0 & $\pi$ & $\pi$ & 0 & $\pi$ & 0 & $\pi$ \\
4 & 0 & $\pi$ & 0 & $\pi$ & $\pi$ & $\pi$ & 0 \\
12 & $\pi$ & $\pi$ & $\pi$ & $\pi$ & 0 & 0 & 0 \\
23 & 0 & 0 & $\pi$ & $\pi$ & 0 & $\pi$ & $\pi$ \\
\hline
\end{tabular}

Table 1: The dihedral angles $\psi_{i}$ on the segments of the caustic curve bounding classically forbidden regions ABCD. There are two possibilities for segments $\mathrm{B}$ and $\mathrm{D}$, and one for segments $\mathrm{A}$ and $\mathrm{C}$.

The possible values of the $\psi_{i}$ on the caustic curve are summarized in Table 1. The four segments of the caustic curve are identified by the classically forbidden region (ABCD) to which they are adjacent. In segments $A, B$ and $\mathrm{D}$ there are two possibilities, while in segment $\mathrm{C}$ there is only one. In segment A, the first column applies if $J_{12 \text {,max }}=J_{3}+J_{4}$ and the second column otherwise; in segment B, the first column applies if $J_{23, \min }=J_{2}-J_{3}$ or $J_{4}-J_{1}$ and the second column otherwise; and in segment D, the first column applies if $J_{23 \text {, max }}=J_{2}+J_{3}$, and the second column otherwise. Examples of these rules may be seen in Fig. 4. The dihedral angles in a flat tetrahedron such as the ones labeled $\mathrm{Y} \rightarrow \mathrm{X}$ and $\mathrm{X} \rightarrow \mathrm{Z}$ are 0 for interior segments and $\pi$ for segments bounding the outside of the plane figure.

Although some angles $\psi_{i}$ are discontinuous at points XYZW, the Ponzano-Regge phase $\Phi_{\mathrm{PR}}$ is continuous everywhere on the caustic boundary (hence everywhere inside and on the caustic boundary).

The angles $\psi_{i}$ that are $\pi$ on a segment of the caustic curve correspond to the vectors $\mathbf{J}_{i}$ that lie on the outside of the plane figure, as seen in the examples in Fig. 4. That is, they correspond to a set of vectors $\mathbf{J}_{i}$ that sum to zero. But this implies that the sum of the corresponding $j_{i}$ values is an integer,

$$
\nu_{6 j}=\sum_{i}^{\prime} j_{i}=\text { integer }
$$

where the prime means to sum only over $i$ such that $\psi_{i}$ on a segment of the caustic curve has the value $\pi$. For example, from the first column for segment $\mathrm{B}$ in Table 1 we have $j_{1}+j_{3}+j_{12}+j_{23}=$ integer.

In the classically forbidden regions the method of Appendix $\mathrm{A}$ yields vectors $\mathbf{J}_{i}$ whose $(x, y, z)$ components can be labeled as $(r, r, i)$, where $r$ 
means real and $i$ means imaginary. These vectors have real lengths $J_{i}$ that are fixed by (8) and the values of the $j_{i}$. The cross products $\mathbf{J}_{i} \times \mathbf{J}_{j}$ have the form $(i, i, r)$ and also have real lengths, which are twice the real areas of the faces. Dividing by these we obtain complex unit normals to the faces of the form $(i, i, r)$, whose dot products, the cosines of the angles $\psi_{i}$, are real. These cosines lie outside the range $[-1,+1]$, however, indicating that the $\psi_{i}$ are complex. Since the complex inverse cosine function has multiple branches, we must determine the branch.

A first requirement is that branch chosen for $\psi_{i}$ should agree with the value of $\psi_{i}$ as we approach caustic curve, where $\psi_{i}$ is either 0 or $\pi$, depending on $i$ and the region ABCD, as shown in Table 1. If $\psi_{i}=0, \cos \psi_{i}=+1$ on the caustic curve, then $\cos \psi_{i}$ is real and $>1$ in the classically forbidden region. In this case we choose $\psi_{i}=i \bar{\psi}_{i}$, where $\bar{\psi}_{i}=\cosh ^{-1}\left(\cos \psi_{i}\right)$ is real and positive. If $\psi_{i}=\pi, \cos \psi_{i}=-1$ on the caustic curve, then $\cos \psi_{i}$ is real and $<-1$ in the classically forbidden region. In this case we choose $\psi_{i}=\pi+i \bar{\psi}_{i}$, where $\bar{\psi}_{i}=-\cosh ^{-1}\left(-\cos \psi_{i}\right)$ is real and negative. We can summarize these two cases by

$$
\bar{\psi}_{i}=\operatorname{sign}\left(\cos \psi_{i}\right) \cosh ^{-1}\left(\left|\cos \psi_{i}\right|\right) .
$$

In spite of the sign and absolute value functions, $\bar{\psi}_{i}$ is a smooth function of position in any of the four classically forbidden regions.

This procedure allows us to determine which classically forbidden region (ABCD) we are in, for if we note the signs of the $\cos \psi_{i}$, those that are $>1$ indicate angles that vanish on the segment of the caustic curve bordering the region, while those that are $<-1$ indicate angles that become $\pi$ on that segment. The pattern of 0's and $\pi$ 's uniquely identifies the region, as shown by Table 1 .

With these definitions, the imaginary part of the analytic continuation of $\Phi_{\mathrm{PR}}$ is

$$
\bar{\Phi}_{\mathrm{PR}}=\sum_{i} J_{i} \bar{\psi}_{i}
$$

where the sum runs over all six $i$. The quantity $\bar{\Phi}_{\mathrm{PR}}$ vanishes on the caustic curve and becomes real and negative as we move into classically forbidden regions $\mathrm{A}$ or $\mathrm{D}$, or real and positive as we move into regions $\mathrm{B}$ or $\mathrm{C}$. The $6 j$ symbol decays exponentially as we move into any classically forbidden region, a behavior that is captured by $\exp \left(-\left|\bar{\Phi}_{\mathrm{PR}}\right|\right)$ in all regions. Since $\Phi_{\mathrm{PR}}$ has only one sign in any of the four classically forbidden regions, its absolute value is a smooth function in those regions. Finally, the Ponzano-Regge approximation 
in the classically forbidden regions is

$$
\left\{\begin{array}{lll}
j_{1} & j_{2} & j_{12} \\
j_{3} & j_{4} & j_{23}
\end{array}\right\} \approx A_{\mathrm{PR}} \exp \left(-\left|\bar{\Phi}_{\mathrm{PR}}\right|\right)
$$

where the amplitude is given by

$$
A_{\mathrm{PR}}=\frac{(-1)^{\nu_{6 j}}}{2 \sqrt{12 \pi|V|}}
$$

and where $\nu_{6 j}$ is given by (18). The definitions we have made allow a single formula to be written down for all four regions, but it is easy to write four formulas for the four regions without the use of sign or absolute value functions.

\subsection{The Phase Space of the $6 j$-symbol}

The phase space of the $6 j$-symbol is the set of all closed figures that can be obtained by placing $\mathbf{J}_{i}, i=1,2,3,4$ end-to-end, for fixed values of the lengths of these vectors, modulo proper rotations. That is, the vectors must satisfy (2). This is the point of view of Kapovich and Millson[16], that recently has been further developed by Charles 13. This space can also be derived by symplectic reduction[17] from a model of four independent angular momenta built around Schwinger's[18] oscillators, much as in the treatment of Aquilanti et al 14 of the $3 j$-symbol. In our applications we will think of the lengths $J_{i}, i=1,2,3,4$ as being given by the quantization condition (8) in terms of the fixed quantum numbers $j_{i}, i=1,2,3,4$ appearing in a $6 j$-symbol. For a given closed chain formed by $\mathbf{J}_{i}, i=1,2,3$, 4, we can draw vectors $\mathbf{J}_{12}$ and $\mathbf{J}_{23}$ defined by (3) to obtain a tetrahedron. Thus the phase space can also be thought of as the set of all real tetrahedra, in which the lengths $J_{i}, i=1,2,3,4$ are fixed. The lengths $J_{12}$ and $J_{23}$, however, vary continuously between the limits (10).

All such tetrahedra are generated if we let $J_{12}$ vary from $J_{12 \text {,min }}$ to $J_{12 \text {,max }}$, while for each value of $J_{12}$ we let the dihedral angle $\phi_{12}$ vary from $-\pi$ to $+\pi$. Here $\phi_{12}$ is the interior dihedral angle, illustrated in Fig. 5, that is uniquely defined in the interval $-\pi<\phi_{12} \leq \pi$ by requiring that $\phi_{12}=0, \pi$ correspond to flat tetrahedra, and that $0<\phi_{12}<\pi$ correspond to tetrahedra with positive volume (this is the case illustrated in Fig. 5). It is related to the exterior dihedral angle $\psi_{12}$ used in the Ponzano-Regge formula by 
$\left|\phi_{12}\right|+\psi_{12}=\pi$. The angle $\phi_{12}$ distinguishes tetrahedra related by spatial inversion (i.e., time reversal), while $\psi_{12}$ does not. Similarly, we could generate all real tetrahedra by varying $J_{23}$ and the dihedral angle $\phi_{23}$. The choice of $J_{12}, \phi_{12}$ for this process is arbitrary, but it gives us a definite convention for coordinates on the phase space of the $6 j$-symbol, namely, $\left(J_{12}, \phi_{12}\right)$.

The manifold of such tetrahedra, modulo proper rotations, is a sphere. To see this, define

$$
J_{12, \text { avg }}=\frac{1}{2}\left(J_{12, \max }+J_{12, \min }\right)
$$

and write

$$
K_{z}=J_{12}-J_{12, \text { avg }},
$$

so that $K_{z}$ varies between $-D / 2$ and $+D / 2$ as $J_{12}$ goes from $J_{12 \text {,min }}$ to $J_{12 \text {,max }}$ (see (12)). Then define a polar angle $\theta_{12}$ by

$$
K_{z}=(D / 2) \cos \theta_{12}
$$

and set

$$
\begin{aligned}
& K_{x}=(D / 2) \sin \theta_{12} \cos \phi_{12}, \\
& K_{y}=(D / 2) \sin \theta_{12} \sin \phi_{12},
\end{aligned}
$$

so that $\left(K_{x}, K_{y}, K_{z}\right)$ are Cartesian coordinates on a sphere of radius $D / 2$ with spherical angles $\left(\theta_{12}, \phi_{12}\right)$. The azimuthal angle $\phi_{12}$ on the sphere is the same as the interior dihedral angle in the tetrahedron.

This is the $6 j$-sphere, on which the north pole is $K_{z}=D / 2$ or $J_{12}=$ $J_{12 \text {,max }}$, the south pole is $K_{z}=-D / 2$ or $J_{12}=J_{12 \text {,min }}$, and curves of constant $J_{12}$ in general are small circles $K_{z}=$ const. It is illustrated in Fig. 6, which shows several curves of constant $J_{12}$. Flat configurations correspond to $\phi_{12}=$ 0 or $\pi$, that is, they lie on the $K_{x}-K_{z}$ plane (the great circle $K_{y}=0$ ). The hemisphere $K_{y}>0\left(K_{y}<0\right)$ consists of tetrahedra of positive (negative) volume. Spatial inversion (i.e., time reversal) is a reflection in the plane $K_{y}=0$ (it amounts to $K_{y} \rightarrow-K_{y}$ ).

Any quantity defined in terms of the tetrahedron that is invariant under proper rotations corresponds to a function on the $6 j$-sphere. For example, $J_{23}$ is such a function, as is $\phi_{23}$. Curves of constant $J_{23}$ are illustrated in Fig. 7. The extrema of $J_{23}$ are both flat configurations lying on the great circle $K_{y}=0$, with $J_{23}=J_{23, \text { min }}$ on the semicircle $K_{x}>0$ and $J_{23}=J_{23 \text {, max }}$ on the semicircle $K_{x}<0$. This is apparent from figures such as diagram 
$\mathrm{X}$ in Fig. 4, which illustrates the case $J_{23}=J_{23 \text {,min }}$ and which shows that $\phi_{12}=0($ not $\pi)$ at such a configuration. Two views of the $6 j$-sphere are given in Fig. 7 to show both extrema of $J_{23}$, as well as curves of constant $J_{23}$ for intermediate values. Notice that the curves of constant $J_{23}$ are not small circles. That is because the coordinates we are using on the $6 j$-sphere make the curves of constant $J_{12}$ look simple (they are small circles), but not the curves of constant $J_{23}$. Had we based our coordinates on $J_{23}$ and $\phi_{23}$ instead, the roles would be reversed.

The diagrams in Fig. 7 were created in the following way. We set up a grid of coordinates $\left(J_{12}, \phi_{12}\right)$ by letting $J_{12}$ vary between $J_{12 \text {,min }}$ and $J_{12 \text {,max }}$, and for each value of $J_{12}$, letting $\phi_{12}$ vary between $\pm \pi$. For each value of $J_{12}$ at $\phi_{12}=0$, we set up the corresponding flat tetrahedron, such as the diagrams labelled $\mathrm{Y} \rightarrow \mathrm{X}$ and $\mathrm{X} \rightarrow \mathrm{Z}$ in Fig. 4, both of which have $\phi_{12}=0$. For other values of $\phi_{12}$, we rotate the triangle 1-2-12 about the axis defined by $\mathbf{J}_{12}$ by angle $\phi_{12}$, using the right-hand rule, while holding triangle $3-4-12$ fixed. This is the "butterfly" motion of the tetrahedron associated with the axis $\mathbf{J}_{12}$. This creates a tetrahedron of the desired dihedral angle $\phi_{12}$. Then we compute $J_{23}$ for that tetrahedron. In this way, we set up an array of $J_{23}$ values on the grid. Finally, we draw the contour lines of $J_{23}$ for this grid, and plot them on the surface of the sphere.

There are two ways to compute Poisson brackets on the $6 j$-sphere. First, let $F$ and $G$ be two functions of the $\mathbf{J}_{i}, i=1,2,3,4$. Then the Poisson bracket is the usual one in classical mechanics for a set of independent angular momenta,

$$
\{F, G\}=\sum_{i=1}^{4} \mathbf{J}_{i} \cdot\left(\frac{\partial F}{\partial \mathbf{J}_{i}} \times \frac{\partial G}{\partial \mathbf{J}_{i}}\right) .
$$

For example, any function of $\mathbf{J}_{1}$ and $\mathbf{J}_{2}$ has vanishing Poisson bracket with any function of $\mathbf{J}_{3}$ and $\mathbf{J}_{4}$.

Hamilton's equations can be expressed in terms of Poisson brackets. Let $H$ be a Hamiltonian with evolution parameter (the "time") $\lambda$, and let $F$ be any function of $\mathbf{J}_{i}, i=1,2,3,4$. Then the rate of change of $F$ along the orbits of $H$ is

$$
\frac{d F}{d \lambda}=\{F, H\}
$$

For example, if we take $H=J_{12}=\left|\mathbf{J}_{1}+\mathbf{J}_{2}\right|$ and $F=$ some component of 
one of the $\mathbf{J}_{i}$, we find

$$
\frac{d \mathbf{J}_{i}}{d \lambda}=\left\{\begin{aligned}
\mathbf{e}_{12} \times \mathbf{J}_{i}, & i=1,2 \\
0, & i=3,4
\end{aligned}\right.
$$

where $\mathbf{e}_{12}$ is the unit vector in the direction $\mathbf{J}_{12}$. The $\lambda$-evolution is a rotation of vectors $\mathbf{J}_{1}$ and $\mathbf{J}_{2}$ about the axis $\mathbf{e}_{12}$, while $\mathbf{J}_{3}$ and $\mathbf{J}_{4}$ remain fixed. This is the "butterfly" motion mentioned above, and $\lambda$ is the angle. If the initial conditions are chosen so that $\lambda=0$ when $\phi_{12}=0$, then $\lambda=\phi_{12}$. On the $6 j$-sphere the orbits of $J_{12}$ are the curves $J_{12}=$ const, the small circles seen in Fig. 6.

Similarly, $J_{23}$ generates another butterfly motion, in which vectors $\mathbf{J}_{2}$ and $\mathbf{J}_{3}$ rotate about the axis $\mathbf{e}_{23}$ by the right-hand rule, with dihedral angle $\phi_{23}$ as the parameter of evolution. Vectors $\mathbf{J}_{1}$ and $\mathbf{J}_{4}$ remain fixed during this motion. The orbits on the $6 j$-sphere are curves of constant $J_{23}$, some examples of which are illustrated in Fig. 7.

Since a Hamiltonian and its evolution parameter are canonically conjugate variables, it follows that $\left(J_{12}, \phi_{12}\right)$ are canonically conjugate variables on the $6 j$-sphere. Since $J_{12}$ differs from $K_{z}$ by a constant, we can equally well use $\left(K_{z}, \phi_{12}\right)$. Thus another way to compute the Poisson bracket of any two functions defined on the $6 j$-sphere is

$$
\{F, G\}=\frac{\partial F}{\partial \phi_{12}} \frac{\partial G}{\partial J_{12}}-\frac{\partial F}{\partial J_{12}} \frac{\partial G}{\partial \phi_{12}} .
$$

This only applies to rotationally invariant functions, while (27) can be used for any functions of the $\mathbf{J}_{i}, i=1,2,3,4$. Also, expressing functions in terms of $J_{12}$ and $\phi_{12}$ is usually difficult, so in practice (27) is more useful than (30).

But (30) does show that the area of a closed curve on the $6 j$-sphere can be computed as

$$
\text { Area }=\oint J_{12} d \phi_{12}
$$

with due attention to the singularities of the $\left(J_{12}, \phi_{12}\right)$ coordinates (there is no global symplectic 1 -form on the sphere). The area is $D / 2$ times the solid angle subtended by the closed loop. This is not the area on the surface of a sphere of radius $D / 2$ in $\left(K_{x}, K_{y}, K_{z}\right)$ space, computed by Euclidean geometry, which would be $(D / 2)^{2}$ times the solid angle, but it is the correct measure of area from the standpoint of semiclassical mechanics.

In particular, the total area of the sphere is $(4 \pi)(D / 2)=2 \pi D$, or $D$ Planck cells of area $2 \pi$ each (it would be $2 \pi \hbar$ in ordinary units). This is 
what we expect for a semiclassical phase space representing the subspace $Z$ of the Hilbert space of four angular momenta, which contains $D$ quantum states.

Moreover, the Bohr-Sommerfeld rules say that the quantized values of $J_{12}$ should be given by orbits $J_{12}=$ const whose area is $\left(n+\frac{1}{2}\right)(2 \pi)$. On the sphere there is no way to distinguish the interior and the exterior of a loop, but the Bohr-Sommerfeld rule is the same in either case since the total area of the sphere is an integer times $2 \pi$. Since the small circle $J_{12}=$ const encloses area $(2 \pi)\left(J_{12}-J_{12 \text {,min }}\right)$ about the south pole, the quantized orbits are those for which $J_{12}=J_{12 \text {,min }}+\frac{1}{2}+$ integer. By (8) this gives the exact quantized values of $j_{12}$, as indicated by (4). The minimum and maximum quantized values of $J_{12}$ are one half unit away from the values at the south and north poles, respectively, corresponding to the one-half unit margin between the quantized spots in Fig. 3 and the bounding values of $J_{12}$. Figure 6 illustrates the quantized orbits of $J_{12}$ for the case $D=5$, numbered 0 to 4 as $J_{12}$ increases (orbit 0 lies close to the south pole and cannot be seen in the figure).

Similarly, the quantized orbits of $J_{23}$ are those satisfying $J_{23}=J_{23 \text {,min }}+$ $\frac{1}{2}+$ integer. These are labeled 0 to 4 in Fig. 7, for the same case $(D=$ 5) illustrated in Fig. 6. The dihedral angle $\phi_{23}$ is an angle parameterizing position along the curves $J_{23}=$ const, although it is not an azimuthal angle in $\left(K_{x}, K_{y}, K_{z}\right)$ space.

But the logic that leads to the conclusion that $\left(J_{12}, \phi_{12}\right)$ are canonical coordinates on the sphere applies also to $\left(J_{23}, \phi_{23}\right)$, so there is a canonical transformation connecting $\left(J_{12}, \phi_{12}\right)$ and $\left(J_{23}, \phi_{23}\right)$. According to Miller's theory [19], the $F_{4}$-type generating function of this canonical transformation is the phase of the semiclassical matrix element $\left\langle j_{12} \mid j_{23}\right\rangle$. (We follow Goldstein's 20] conventions for classifying generating functions.)

Miller's theory leads to difficult integrals in cases like this (it certainly does for the $3 j$-symbol), and it has never been carried through for the $6 j$ symbol, as far as we know. But it is certain that the $F_{4}$-type generating function that would result would be the Ponzano-Regge phase $\Phi_{\mathrm{PR}}$, to within an additive constant. Moreover, Miller's theory shows that the amplitude determinant in the Ponzano-Regge formula is given by

$$
A_{\mathrm{PR}}=\left|\frac{\partial^{2} \Phi_{\mathrm{PR}}}{\partial J_{12} \partial J_{23}}\right|^{1 / 2},
$$

to within a multiplicative constant. In this formula, the lengths $J_{i}, i=$ 
1, 2, 3, 4 are considered fixed, and the dihedral angles $\psi_{i}$ that appear in (15) are considered functions of all six lengths $J_{i}$.

The Ponzano-Regge amplitude was first derived by Wigner[21], who had the intuition that the probability in making a measurement of $j_{23}$ for given value of $j_{12}$ should be uniformly distributed in the angle $\phi_{12}$. That this is so follows from standard semiclassical theory and the fact that $\phi_{12}$ is conjugate to $J_{12}$. This amplitude is also inversely proportional to the square root of a Poisson bracket,

$$
\left\{J_{23}, J_{12}\right\}=\frac{\mathbf{J}_{1} \cdot\left(\mathbf{J}_{2} \times \mathbf{J}_{3}\right)}{J_{12} J_{23}}=\frac{6 V}{J_{12} J_{23}}
$$

which was computed using (27). The volume factor $V$ is the part of the amplitude that was obtained by Wigner, while the factor $J_{12} J_{23}$, when replaced by $\left(j_{12}+\frac{1}{2}\right)\left(j_{23}+\frac{1}{2}\right)$, is needed to convert from the unitary matrix element $\left\langle j_{12} \mid j_{23}\right\rangle$ to the $6 j$-symbol (see Edmonds[1] Eq. (6.2.10)). The use of Poisson brackets for computing amplitude determinants is discussed in Littlejohn[22] and in Aquilanti et al[14].

The relation between Fig. 3 and the phase space of the $6 j$-symbol is the following. A point inside the square of Fig. 3 specifies values of $J_{12}$ and $J_{23}$ that are allowed by the inequalities (9). These in turn specify two curves in the phase space, one of constant $J_{12}$ and the other of constant $J_{23}$. If these curves intersect, as in part (a) of Fig. 8, then we are in the classically allowed region. In that case, the intersection points, labeled $P$ and $Q$ in the figure, are the stationary phase points of the semiclassical evaluation of the matrix element $\left\langle j_{12} \mid j_{23}\right\rangle$. These points represent two tetrahedra that are mirror images of each other (they are related by time reversal). The total semiclassical matrix element (the Ponzano-Regge formula) is a sum of contributions from these two tetrahedra, which are complex conjugates of each other. Thus, the semiclassical matrix element is real.

If the two curves do not intersect, as in part (b) of Fig. 8, then we are in the classically forbidden region. Both curves are manifolds of real tetrahedra, one with a fixed value of $J_{12}$, the other of $J_{23}$, but since they do not intersect, there is no real tetrahedron that simultaneously has both given values of $J_{12}$ and $J_{23}$. In this case the analytic continuations of the curves into complex phase space (a complexified sphere) do intersect, and these intersections represent the stationary phase points in the classically forbidden region. We make no attempt to sketch the complexified phase space, however. 
In addition to its interpretation as a generating function, the phase of semiclassical matrix elements such as $\left\langle j_{12} \mid j_{23}\right\rangle$ is geometrically one half the area enclosed by the intersection of the quantized classical orbits in phase space[22]. (The relative phase between the two branches of the WKB solution is the area, but this is shared between two exponentials to create a cosine term. Thus the argument of the cosine is one half the area.) In part (a) of Fig. 8 the area in question is the shaded region (a "lune").

If the two curves $J_{12}=$ const and $J_{23}=$ const are tangent, then we are at a caustic. A caustic implies a flat tetrahedron of zero volume, so such tangencies can occur only in the plane $K_{y}=0$.

The different types of caustics than can occur are illustrated in Fig. 9. Part A of Fig. 9 is obtained from the curves of Fig. 8 by adjusting the $J_{12}$ or $J_{23}$ values to create a tangency. As expected, it lies between the classically allowed region (part (a) of Fig. 8) and the classically forbidden region (part (b) of Fig. 8). The point of tangency $T$ is the caustic point. From part A of Fig. 9 we move into the classically allowed region if we either decrease $J_{12}$ or increase $J_{23}$. Thus we see that it corresponds to region A of Fig. 3.

If we allow $J_{12}$ in part A of Fig. 9 to decrease, the small circles $J_{12}=$ const sweep down from the north pole through the oval $J_{23}=$ const, passing through the classically allowed region, until a tangency is reached at the lower point of the oval $J_{23}=$ const, where the small circle $J_{12}=$ const is close to the equator. This is another caustic, illustrated in part B of Fig. 9. The shaded area is the continuation of the shaded area in part (a) of Fig. 8, and again $T$ is the caustic point. From this caustic, we pass back into the classically allowed region if either $J_{12}$ increases or $J_{23}$ increases, so we are in region B of Fig. 3.

If from part A of Fig. 9 we allow $J_{23}$ to increase, then the curves $J_{23}=$ const sweep through the small circle $J_{12}=$ const about the north pole, finally reaching another tangency on the other side where $K_{x}<0$. The result is illustrated in part $\mathrm{C}$ of Fig. 9, where again point $T$ is the caustic point. From this caustic we pass back into the classically allowed region if we let either $J_{12}$ or $J_{23}$ decrease, so this corresponds to region C of Fig. 3 ,

As $J_{23}$ increases from its value in part C of Fig. 9, the curve $J_{23}=$ const shrinks down around the point $J_{23}=J_{23, \max }$ on the semicircle $K_{y}=0$, $K_{x}<0$. Then allowing $J_{12}$ to decrease, the small circle around the north pole moves south, passing through the curve $J_{23}=$ const, producing finally a tangency $T$ on the other side, as illustrated in part D of Fig. 9. Now the 
shaded area (twice the Ponzano-Regge phase plus a constant) covers nearly the entire sphere. From this configuration we pass back into a classically allowed region if either $J_{23}$ decreases or $J_{12}$ increases, so we are in region D in Fig. 3.

In the case of an ordinary oscillator with a flat phase space (the plane), the difference in the actions between the two turning points is one half the area of the orbit, and has the form $\left(n+\frac{1}{2}\right) \pi$, where $n$ is an integer. As explained in the introduction, this is a requirement for the existence of a uniform approximation of the Weber function type. The analogous statement for the $6 j$-symbol with the spherical phase space is sometimes true, and sometimes not. A case where it is true is obtained from diagrams A and B of Fig. 9 in which we regard $j_{23}$ fixed and $j_{12}$ variable. As $J_{12}$ decreases from the north pole (its maximum value), we first encounter a caustic of the type A, where the area of the lune is zero. Continuing to decrease $J_{12}$, we pass through the classically allowed region, finally encountering a caustic of the type B (the lower turning point), where the area of the lune is the quantized area of the oval $j_{23}=$ const. This area has the form $\left(n+\frac{1}{2}\right) 2 \pi$, so the differences in the actions at the two turning points is quantized. This implies that the difference in the Ponzano-Regge phases $\Phi_{\mathrm{PR}}$ between the two turning points is also quantized.

A case where the differences in the actions is not quantized and a uniform approximation of the Weber function type does not exist is obtained when $j_{23}$ has a value such as that illustrated in part $\mathrm{C}$ of Fig. 9. In this case, as we let $J_{12}$ decrease from its maximum value at the north pole the first caustic we encounter is of type $\mathrm{C}$, where the area of the lune is the area of the curve $J_{12}=$ const (the shaded area in part $\mathrm{C}$ of the figure). This area is not quantized, since the value of $J_{12}$ at a caustic is not quantized. As $J_{12}$ decreases, we eventually reach the lower caustic of type B, where the area of the lune is the quantized area of the orbit $j_{23}=$ const. Thus, the differences between the areas, one quantized, the other not, is not quantized. A case like this (with caustics of the type B and C) was illustrated in Fig. 1.

We will now show that the $d$-matrices have a phase space and an orbit and caustic structure that are identical, from a topological standpoint, to those of the $6 j$-symbol. 


\section{The $d$-matrices}

\subsection{Quantum Mechanics of the $d$-Matrices}

The $d$-matrices are defined by

$$
d_{m m^{\prime}}^{j}(\beta)=\left\langle m\left|U_{y}(\beta)\right| m^{\prime}\right\rangle,
$$

where $U_{y}(\beta)=\exp \left(-i \beta J_{y}\right)$ is a rotation operator with Euler angle $\beta$ about the $y$-axis, and $|m\rangle$ and $\left|m^{\prime}\right\rangle$ are standard rotation basis states (eigenstates of $J_{z}$ ). To indicate both the operator and the quantum number, we will write these states as $\left|J_{z}: m\right\rangle$ and $\left|J_{z}: m^{\prime}\right\rangle$. By conjugation the rotation operator $U_{y}(\beta)$ rotates the angular momentum vector,

$$
U_{y}(\beta)^{\dagger} \mathbf{J} U_{y}(\beta)=R_{y}(\beta) \mathbf{J}
$$

where $R_{y}(\beta)$ is the $3 \times 3$ rotation matrix for an active rotation about the $y$-axis. We define

$$
\hat{\mathbf{n}}=R_{y}(\beta) \hat{\mathbf{z}}=\left(\begin{array}{ccc}
\cos \beta & 0 & \sin \beta \\
0 & 1 & 0 \\
-\sin \beta & 0 & \cos \beta
\end{array}\right)\left(\begin{array}{l}
0 \\
0 \\
1
\end{array}\right)=\left(\begin{array}{c}
\cos \beta \\
0 \\
\sin \beta
\end{array}\right)
$$

as illustrated in Fig. 10, so that

$$
(\hat{\mathbf{n}} \cdot \mathbf{J}) U_{y}(\beta)\left|J_{z}: m^{\prime}\right\rangle=U_{y}(\beta)(\hat{\mathbf{z}} \cdot \mathbf{J})\left|J_{z}: m^{\prime}\right\rangle=m^{\prime} U_{y}(\beta)\left|J_{z}: m^{\prime}\right\rangle
$$

where we use (35) and

$$
\hat{\mathbf{n}} \cdot\left[R_{y}(\beta) \mathbf{J}\right]=\left[R_{y}(\beta)^{-1} \hat{\mathbf{n}}\right] \cdot \mathbf{J}=\hat{\mathbf{z}} \cdot \mathbf{J}=J_{z} .
$$

Therefore $U_{y}(\beta)\left|J_{z}: m^{\prime}\right\rangle$ is an eigenstate of $\hat{\mathbf{n}} \cdot \mathbf{J} \equiv J_{n}$ with eigenvalue $m^{\prime}$, and we will write

$$
U_{y}(\beta)\left|J_{z}: m^{\prime}\right\rangle=\left|J_{n}: m^{\prime}\right\rangle
$$

so that

$$
d_{m m^{\prime}}^{j}(\beta)=\left\langle J_{z}: m \mid J_{n}: m^{\prime}\right\rangle .
$$

In this way the $d$-matrix is written as a unitary matrix element connecting the eigenstates of two different operators. This is the starting point for Miller's [19] theory of semiclassical matrix elements, as well as our own 22, 14] treatments of the same subject. 


\subsection{Classical and Semiclassical Mechanics of the $d$-ma- trices}

References on the semiclassical approximation for the $d$-matrices include Brussaard and Tolhoek[23], Ponzano and Regge[10], Braun et al[24] and Sokolovski and Connor [25]. In the following we emphasize geometrical aspects of the problem not covered by these authors.

The classical phase space for $d_{m m^{\prime}}^{j}(\beta)$ is a sphere ("the $d$-sphere") in angular momentum space of radius $|\mathbf{J}|=J$, where

$$
J=j+\frac{1}{2}
$$

The area of a loop on the surface of the sphere is given by

$$
\text { Area }=\oint J_{z} d \phi
$$

where $\phi$ is the azimuthal angle, again with due consideration of the singularities of the coordinates $\left(\phi, J_{z}\right)$. That is, if the loop subtends solid angle $\Omega$, then the area is $J \Omega$ (not $J^{2} \Omega$, as in Euclidean geometry). The total area of the sphere is therefore $4 \pi J=(2 j+1)(2 \pi)$, that is, the sphere consists of $2 j+1$ Planck cells, corresponding to the $2 j+1$ basis states $\left|J_{z}: m\right\rangle$ or $\left|J_{n}: m^{\prime}\right\rangle$. Curves of constant $\hat{\mathbf{z}} \cdot \mathbf{J}=J_{z}$ and $\hat{\mathbf{n}} \cdot \mathbf{J}=J_{n}$ are small circles centered on the axes $\hat{\mathbf{z}}$ and $\hat{\mathbf{n}}$, respectively, as illustrated in Fig. 11.

The Poisson bracket of two functions $F$ and $G$ of $\mathbf{J}$ is (27) with a single term in the sum,

$$
\{F, G\}=\mathbf{J} \cdot\left(\frac{\partial F}{\partial \mathbf{J}} \times \frac{\partial G}{\partial \mathbf{J}}\right),
$$

or, equivalently, for functions of $\left(\phi, J_{z}\right)$,

$$
\{F, G\}=\frac{\partial F}{\partial \phi} \frac{\partial G}{\partial J_{z}}-\frac{\partial F}{\partial J_{z}} \frac{\partial G}{\partial \phi} .
$$

For example, using (43) we find that Hamilton's equations for Hamiltonian $J_{z}=\hat{\mathbf{z}} \cdot \mathbf{J}$ with evolution parameter $\lambda$ are

$$
\frac{d \mathbf{J}}{d \lambda}=\hat{\mathbf{z}} \times \mathbf{J} .
$$

The motion is a rotation about the $z$-axis, so the orbits are the small circles $J_{z}=$ const, as expected. The parameter of the orbit is $\lambda=\phi$, so $\phi$ and $J_{z}$ are 
conjugate variables, as indicated in (44). Similarly, $J_{n}$ generates rotations about the axis $\hat{\mathbf{n}}$.

Let $(\theta, \phi)$ be the usual spherical coordinates referred to the axis $\hat{\mathbf{z}}$, and let $\left(\theta^{\prime}, \phi^{\prime}\right)$ be an alternative set referred to the axis $\hat{\mathbf{n}}$. That is, the $\left(\theta^{\prime}, \phi^{\prime}\right)$ coordinates of a point $(x, y, z)$ are the same as the $(\theta, \phi)$ coordinates of the inverse rotated point $R_{y}(\beta)^{-1}(x, y, z)$. Thus the coordinate transformation $(\theta, \phi) \rightarrow\left(\theta^{\prime}, \phi^{\prime}\right)$ is specified by

$$
\begin{aligned}
\sin \theta \cos \phi & =\cos \beta \sin \theta^{\prime} \cos \phi^{\prime}+\sin \beta \cos \theta^{\prime}, \\
\sin \theta \sin \phi & =\sin \theta^{\prime} \sin \phi^{\prime}, \\
\cos \theta & =-\sin \beta \sin \theta^{\prime} \cos \phi^{\prime}+\cos \beta \cos \theta^{\prime} .
\end{aligned}
$$

The azimuthal angle $\phi^{\prime}$ is conjugate to $J_{n}$, so both $\left(\phi, J_{z}\right)$ and $\left(\phi^{\prime}, J_{n}\right)$ are canonical coordinates on the sphere. The $F_{4}$-type generating function of the canonical transformation between these coordinates is the phase of the semiclassical approximation to the $d$-matrices, according to Miller's theory. This aspect of the problem has been developed by Sokolovski and Connor 25].

The classical observables $J_{z}$ and $J_{n}$ are functions on the $d$-sphere that vary continuously between the limits,

$$
-J \leq J_{z}, J_{n} \leq+J
$$

The quantized orbits of $J_{z}$ and $J_{n}$ are those enclosing $n+\frac{1}{2}$ Planck cells where $n$ is an integer. This implies $J_{z}=m$ and $J_{n}=m^{\prime}$ with the usual rules for quantum numbers $m$ and $m^{\prime}$,

$$
-j \leq m, m^{\prime} \leq+j
$$

in integer steps. Thus the maximum and minimum values of $m$ and $m^{\prime}$ lie one half unit away from the maximum and minimum values of the classical observables $J_{z}$ and $J_{n}$, as illustrated in Fig. 12. This figure may be compared to Fig. 3 for the $6 j$-symbol. See also Fig. 1 of Braun et al[24].

When the $J_{z}$-orbit and the $J_{n}$-orbit intersect one another as in part (a) of Fig. 11, then we are in the classically allowed region of the $d$-matrices. There are generically two intersection points related by a reflection in the plane $J_{y}=0$, marked by unit vectors from the origin $\hat{\mathbf{a}}$ and $\hat{\mathbf{a}}^{\prime}$ in Fig. 13 . We concentrate on intersection $\hat{\mathbf{a}}$, for which $J_{y}>0$; at the other intersection $\hat{\mathbf{a}}^{\prime}$ we have $J_{y}<0$. In the coordinate systems $(\theta, \phi),\left(\theta^{\prime}, \phi^{\prime}\right)$, the $\theta$ and $\theta^{\prime}$ 
coordinates of intersection $\hat{\mathbf{a}}$ are given by

$$
\cos \theta=\frac{J_{z}}{J}=\frac{m}{j+\frac{1}{2}}, \quad \cos \theta^{\prime}=\frac{J_{n}}{J}=\frac{m^{\prime}}{j+\frac{1}{2}},
$$

where $J_{z}$ and $J_{n}$ label the two small circles and where the second form applies if $J, J_{z}$ and $J_{n}$ take on their quantized values. As for the $\phi$ and $\phi^{\prime}$ coordinates of intersection point $\hat{\mathbf{a}}$, they can be obtained by solving (46), assuming $\theta, \theta^{\prime}$ and $\beta$ are given. This gives

$$
\cos \phi=\frac{\cos \theta^{\prime}-\cos \beta \cos \theta}{\sin \beta \sin \theta}, \quad \cos \eta=\frac{\cos \theta-\cos \beta \cos \theta^{\prime}}{\sin \beta \sin \theta^{\prime}},
$$

where we write $\eta=\pi-\phi^{\prime}$ as illustrated in Fig. 13, Equations (50) uniquely determine $\phi$ and $\eta$ in the interval $[0, \pi]$ (assuming that the $J_{z^{-}}$and $J_{n^{-}}$orbits actually intersect).

Figure 13 draws attention to the spherical triangle defined by $\hat{\mathbf{z}}, \hat{\mathbf{n}}$ and $\hat{\mathbf{a}}$, whose sides are arcs of great circles subtending angles $\theta, \theta^{\prime}$ and $\beta$. Equations (50) are the law of cosines for spherical triangles, applied to the interior angles $\phi$ and $\eta$, as shown in the figure. As for the third interior angle, we define $\kappa$ as the opening angle of the lune (the shaded area), as illustrated in the figure. Then it is easy to show that the third interior angle of the spherical triangle at vertex $\hat{\mathbf{a}}$ is $\pi-\kappa$. For this angle the law of cosines gives

$$
\cos (\pi-\kappa)=\frac{\cos \beta-\cos \theta \cos \theta^{\prime}}{\sin \theta \sin \theta^{\prime}}=-\cos \kappa,
$$

determining $\kappa$ also uniquely in the interval $[0, \pi]$.

We define $\Phi_{d}$ as one half of the area of the shaded lune seen in Fig. 13, which is also the $F_{4}$-type generating function of the transformation $(\theta, \phi) \rightarrow$ $\left(\theta^{\prime}, \phi^{\prime}\right)$ (Sokolovski and Connor[25]). Then we have

$$
\begin{aligned}
\Phi_{d} & =J\left(\kappa-\phi \cos \theta-\eta \cos \theta^{\prime}\right) \\
& =J \kappa-J_{z} \phi-J_{n} \eta=\left(j+\frac{1}{2}\right) \kappa-m \phi-m^{\prime} \eta,
\end{aligned}
$$

where the final form applies if $J, J_{z}$ and $J_{n}$ take on their quantized values. The result is the sum of angular momentum quantum numbers times dihedral angles, that is, the interior angles of the spherical triangle formed by $(\hat{\mathbf{z}}, \hat{\mathbf{n}}, \hat{\mathbf{a}})$ are also the interior dihedral angles of the tetrahedron or parallelepiped formed by those vectors. The analogy with the Ponzano-Regge 
formula is more transparent if we use the exterior dihedral angles $\kappa, \pi-\phi$ and $\pi-\eta$, so that

$$
\Phi_{d}=\left(j+\frac{1}{2}\right) \kappa+m(\pi-\phi)+m^{\prime}(\pi-\eta)-\left(m+m^{\prime}\right) \pi,
$$

in which the first three terms look like the sum (15), while the final term just produces a phase factor $(-1)^{m+m^{\prime}}$ in the asymptotic formula.

It is straightforward to prove (52) by elementary geometry, but another proof, based on symplectic reduction of Schwinger's oscillator model of angular momentum[18] (essentially the Hopf fibration), leads to the following generalization. Let a polygon on the unit sphere be specified by vertices $\left(\hat{\mathbf{v}}_{1}, \ldots, \hat{\mathbf{v}}_{n}\right)$ connected by arcs of small circles, where the small circle proceeding from $\hat{\mathbf{v}}_{i}$ to $\hat{\mathbf{v}}_{i+1}$ is obtained by rotating $\hat{\mathbf{v}}_{i}$ about axis $\hat{\mathbf{n}}_{i}$ by angle $\phi_{i}$, using the right-hand rule. Also, let $\kappa_{i}$ be the interior angle between the two small circles meeting at $\hat{\mathbf{v}}_{i}$. Then the solid angle of the interior of the polyhedron, defined as the region to the left as we move along the small circles, is

$$
\Omega=2 \pi-\sum_{i=1}^{n}\left[\left(\pi-\kappa_{i}\right)+\left(\hat{\mathbf{v}}_{i} \cdot \hat{\mathbf{n}}_{i}\right) \phi_{i}\right] .
$$

Special cases of this formula include the solid angle of a spherical triangle (with sides that are great circles), $\Omega=\kappa_{1}+\kappa_{2}+\kappa_{3}-\pi$, and (52), for which $n=2, \kappa_{1}=\kappa_{2}=\kappa$, and $\Phi_{d}=\Omega / 2$. Equation (54) can also be derived as a special case of the Gauss-Bonnet theorem.

The spherical triangle formed by $(\hat{\mathbf{z}}, \hat{\mathbf{n}}, \hat{\mathbf{a}})$ plays another role. We define $V_{d}$ as the volume of the parallelepiped spanned by these three vectors, which can be written in a variety of ways,

$$
\begin{aligned}
V_{d} & =(\hat{\mathbf{z}} \times \hat{\mathbf{n}}) \cdot \hat{\mathbf{a}}=\sin \beta \sin \theta \sin \phi \\
& =\sin \beta \sin \theta^{\prime} \sin \eta=\sin \theta \sin \theta^{\prime} \sin \kappa
\end{aligned}
$$

where we use the law of sines for the final three expressions. One of these equalities is equivalent to the $y$-component of (46). The square of $V_{d}$ is the determinant of the Gram matrix formed by vectors $(\hat{\mathbf{z}}, \hat{\mathbf{n}}, \hat{\mathbf{a}})$,

$$
\begin{aligned}
V_{d}^{2} & =\operatorname{det}\left(\begin{array}{ccc}
\hat{\mathbf{z}} \cdot \hat{\mathbf{z}} & \hat{\mathbf{z}} \cdot \hat{\mathbf{n}} & \hat{\mathbf{z}} \cdot \hat{\mathbf{a}} \\
\hat{\mathbf{n}} \cdot \hat{\mathbf{z}} & \hat{\mathbf{n}} \cdot \hat{\mathbf{n}} & \hat{\mathbf{n}} \cdot \hat{\mathbf{a}} \\
\hat{\mathbf{a}} \cdot \hat{\mathbf{z}} & \hat{\mathbf{a}} \cdot \hat{\mathbf{n}} & \hat{\mathbf{a}} \cdot \hat{\mathbf{a}}
\end{array}\right)=\operatorname{det}\left(\begin{array}{ccc}
1 & \cos \beta & \cos \theta \\
\cos \beta & 1 & \cos \theta^{\prime} \\
\cos \theta & \cos \theta^{\prime} & 1
\end{array}\right) \\
& =1+2 \cos \beta \cos \theta \cos \theta^{\prime}-\cos ^{2} \beta-\cos ^{2} \theta-\cos ^{2} \theta^{\prime} .
\end{aligned}
$$


Since we are working with the volume of the parallelepiped instead of the volume of the tetrahedron, there is no factor of 6 in (55) or of $6^{2}=36$ in (56). The volume $V_{d}$ appears in the amplitude of the asymptotic formulas (57) and (64).

Including all the details (Maslov indices, phase conventions, etc), the asymptotic expression for the $d$-matrix in the classically allowed region is

$$
d_{m m^{\prime}}^{j}(\beta)=A_{d} \cos \left(\Phi_{d}-\frac{\pi}{4}\right)
$$

where the amplitude is

$$
A_{d}=\frac{(-1)^{j-m^{\prime}}}{\sqrt{(\pi / 2) J\left|V_{d}\right|}} .
$$

According to Ref. 22] the amplitude of the WKB approximation for the $d$-matrix should be proportional to the inverse square root of the Poisson bracket $\left\{J_{z}, J_{n}\right\}$, evaluated at the intersection between the two orbits, $\mathbf{J}=$ Jâ. Indeed, using (43), we see that it is:

$$
\begin{aligned}
\left\{J_{z}, J_{n}\right\} & =(\hat{\mathbf{z}} \times \hat{\mathbf{n}}) \cdot \mathbf{J}=J V_{d}=\sin \beta J_{y} \\
& =J \sin \beta \sin \theta \sin \phi .
\end{aligned}
$$

The caustics of the $d$-matrices occur when the small circles $J_{z}=$ const and $J_{n}=$ const are tangent, or, equivalently, when the vectors $(\hat{\mathbf{z}}, \hat{\mathbf{n}}, \hat{\mathbf{a}})$ are linearly dependent so that $V_{d}=0$. Multiplying (56) by $J^{2}$, using (49) and setting the result to zero gives us the equation of the caustic in $J_{z^{-}} J_{n}$ space,

$$
J_{z}^{2}+J_{n}^{2}-2 J_{z} J_{n} \cos \beta-J^{2} \sin ^{2} \beta=0,
$$

an ellipse whose axes are oriented $45^{\circ}$ to the $J_{z^{-}} J_{n}$ axes, and whose semimajor and semiminor axes are $\sqrt{2} \cos (\beta / 2), \sqrt{2} \sin (\beta / 2)$. An example is illustrated in Fig. 12, see also Fig. 1 of Ref. [24]. The ellipse touches the boundary defined by the classical limits (47) at four points, creating four classically forbidden regions labeled ABCD in Fig 12. The square of the volume $V_{d}^{2}$ is negative in the classically forbidden regions, and $V_{d}$ itself is imaginary there.

Another point of view on the caustics is to hold $J_{z}$ and $J_{n}$ fixed, thereby fixing the sizes of the two small circles, and to vary $\beta$, which moves the position of the small circle $J_{n}=$ const. Then the small circles are tangent at the turning points $\beta=\beta_{1}$ or $\beta_{2}$, where $0 \leq \beta_{1} \leq \beta_{2} \leq \pi$, and where

$$
\beta_{1}=\left|\theta-\theta^{\prime}\right|, \quad \beta_{2}=\min \left(\theta+\theta^{\prime}, 2 \pi-\theta-\theta^{\prime}\right) .
$$


The classically allowed region is $\beta_{1} \leq \beta \leq \beta_{2}$, while the two classically forbidden regions are $0 \leq \beta \leq \beta_{1}$ and $\beta_{2} \leq \beta \leq \pi$.

The four types of tangencies of the two small circles are illustrated in Fig. 14. In all four parts of the figure, $T$ is the caustic point (the point of tangency). In part $\mathrm{A}$ we are at the upper turning point $\beta=\beta_{2}$, because if $\beta$ decreases we obtain two intersection points and are in the classically allowed region. In fact, this is the case $\beta_{2}=\theta+\theta^{\prime}<\pi$. Or if we hold $\beta$ fixed but decrease either $J_{z}$ or $J_{n}$, again we enter the classically allowed region, since one or the other of the two small circles expands and the tangency develops into two intersection points. Thus part A of Fig. 14 corresponds to the corner $\mathrm{A}$ of Fig. 12. In part B of Fig. 14 we are at the lower turning point $\beta=\beta_{1}=\theta-\theta^{\prime}>0$, since if $\beta$ increases we move into the classically allowed region. The same happens if we hold $\beta$ fixed and either increase $J_{z}$ or decrease $J_{n}$, so this corresponds to corner B of Fig. 12, In part C of Fig. 14 we are at the lower turning point $\beta=\beta_{1}=\theta^{\prime}-\theta>0$, which corresponds to corner $\mathrm{C}$ of Fig. 12 since we enter the classically allowed region if either $J_{n}$ increases or $J_{z}$ decreases. Finally, in part D of Fig. 14 we are at the upper turning point $\beta_{2}=2 \pi-\theta-\theta^{\prime}<\pi$, which corresponds to corner D of Fig. 12 since we enter the classically allowed region if either $J_{z}$ or $J_{n}$ increases.

The four types of tangencies of orbits for the $6 j$-symbol, illustrated in Fig. 9, are topologically identical to the four types for the $d$-matrices, illustrated in Fig. 14. Similarly, the four classically forbidden regions of the $6 j$-symbol, illustrated in Fig. 3, are in one-to-one correspondence with the four classically forbidden regions of the $d$-matrices, illustrated in Fig. 12. Comparing Figs. 3 and 12, we see that the labelings of the corners by ABCD are not the same; but this is because the point on the $d$-sphere of maximum $J_{n}$, namely, the point in the direction $\hat{\mathbf{n}}$, corresponds to the point on the $6 j$-sphere of minimum $J_{23}$. If the $J_{23}$ axis in Fig. 3 had been drawn increasing downward instead of upward, the labels on all four corners (classically forbidden regions) of both Fig. 3 and Fig. 12 would coincide.

Referring to Fig. 12, if we hold $\beta$ fixed and vary $J_{z}$ or $J_{n}$, moving from the interior of the ellipse (the classically allowed region) to the boundary (the caustic), then all the angles $\phi, \eta$ and $\kappa$ approach either 0 or $\pi$, depending on which segment ABCD of the boundary (the caustic curve) we approach. The values of these angles on the caustics are summarized in Table 2. For uniformity of notation, we write $\alpha_{i}, i=1,2,3$ for $\kappa, \phi$ and $\eta$, as indicated in the table, and similarly we write $k_{i}, i=1,2,3$ for $j,-m,-m^{\prime}$, where the signs are the same as in the three terms of the expression (52) for $\Phi_{d}$. Also 


\begin{tabular}{|c|c|c||c|c|c|c|}
\hline$i$ & $\alpha_{i}$ & $k_{i}$ & $\mathrm{~A}$ & $\mathrm{~B}$ & $\mathrm{C}$ & $\mathrm{D}$ \\
\hline \hline 1 & $\kappa$ & $j$ & 0 & $\pi$ & $\pi$ & 0 \\
2 & $\phi$ & $-m$ & 0 & 0 & $\pi$ & $\pi$ \\
3 & $\eta$ & $-m^{\prime}$ & 0 & $\pi$ & 0 & $\pi$ \\
\hline \hline & & $\nu_{d}$ & 0 & $j-m^{\prime}$ & $j-m$ & $-m-m^{\prime}$ \\
\hline
\end{tabular}

Table 2: Values of the angles $\kappa, \phi$ and $\eta$ on caustics of type ABCD, also integer $\nu_{d}$ for four caustic types.

shown in the table is the integer $\nu_{d}$ for the four classically forbidden regions, defined by

$$
\nu_{d}=\sum_{i}^{\prime} k_{i}=\text { integer, }
$$

where the sum is only taken over $i$ such that $\alpha_{i}=\pi$. The definition is similar to that of $\nu_{6 j}$ in (18), and used for a similar purpose, that is, the asymptotic form of the $d$-matrices in the classically forbidden regions carries a phase $(-1)^{\nu_{d}}$, effectively due to the analytic continuation of $\Phi_{d}$.

The angles $\alpha_{i}$ are extended into the classically forbidden region in a manner exactly like that used for the $\psi_{i}$ in the case of the $6 j$-symbol, as explained below (18). That is, if $\alpha_{i}=0$ on the segment of the caustic curve adjacent to a given classically forbidden region, then we define $\alpha_{i}=i \bar{\alpha}_{i}$, where $\bar{\alpha}_{i}=\cosh ^{-1}\left(\cos \alpha_{i}\right)$ is real and positive; while if $\alpha_{i}=\pi$ on the caustic curve, then we define $\alpha_{i}=\pi+i \bar{\alpha}_{i}$, so that $\bar{\alpha}_{i}=-\cosh ^{-1}\left(-\cos \alpha_{i}\right)$ is real and negative. In the classically forbidden regions, the quantities $\cos \alpha_{i}$, given by (50) and (51), lie outside the interval $[-1,-1]$.

We now define a quantity related to the analytic continuation of $\Phi_{d}$ into the classically forbidden regions,

$$
\bar{\Phi}_{d}=\sum_{i=1}^{3} k_{i} \bar{\alpha}_{i}=\sum_{i=1}^{3} k_{i} \operatorname{sign}\left(\cos \alpha_{i}\right) \cosh ^{-1}\left|\cos \alpha_{i}\right| .
$$

In spite of the absolute value and sign functions, $\bar{\Phi}_{d}$ is smooth over any given classically forbidden region. This is important for root finders that rely on smoothness, such as the Newton-Raphson method. The quantity $\bar{\Phi}_{d}$ is zero on the caustic boundary, and real and negative as we move into classically forbidden regions $\mathrm{B}$ and $\mathrm{C}$, and real and positive as we move into classically forbidden regions $\mathrm{A}$ and $\mathrm{D}$. These are the same rules as for $\bar{\Phi}_{\mathrm{PR}}$. The $d$ matrices decrease exponentially as we move into any classically forbidden region, a behavior that is captured by $\exp \left(-\left|\bar{\Phi}_{d}\right|\right)$ in all cases. 
Finally, the asymptotic expression for the $d$-matrices in the classically forbidden region is

$$
d_{m m^{\prime}}^{j}=A_{d} \exp \left(-\left|\bar{\Phi}_{d}\right|\right),
$$

where

$$
A_{d}=\frac{(-1)^{j-m^{\prime}+\nu_{d}}}{2 \sqrt{(\pi / 2) J\left|V_{d}\right|}} .
$$

\section{The Uniform Approximation}

\subsection{Remarks on Uniform Semiclassical Approximations}

The traditional method of constructing uniform semiclassical approximations, the "method of comparison equations," is reviewed by Berry and Mount [26], with citations to earlier literature. In this method one takes a one-dimensional Schrödinger equation (a second-order differential equation in $x$ ) and performs a coordinate transformation $X=X(x)$ to create a new Schrödinger equation in $X$ which, after the neglect of terms of order $\hbar^{2}$, becomes a standard, solvable equation. The most common standard or "comparison" equations in practice are the differential equations for Airy or Weber (parabolic cylinder) functions.

Since both the $6 j$-symbols and the $d$-matrices satisfy second-order difference equations, it is likely that a kind of discrete version of the method of comparison equations, along the lines of the discrete WKB theory used by Schulten and Gordon[11] and refined by Braun[15], could be used to construct a uniform approximation for the $6 j$-symbol. We have not constructed our approximation in this way, however, and if we had, it is likely that we would have missed much of the geometry discussed above. Also, that approach would have produced a uniform approximation for the $6 j$-symbol only for a fixed value of $j_{12}$ or $j_{23}$, not over the whole range of both variables as we have done here (it would not have produced a uniform approximation in terms of $d$-matrices).

Hiding slightly beneath the surface of the usual method of comparison equations is a transformation between the phase spaces of the original problem and the standard problem. This transformation is $X=X(x)$ and $P=(d x / d X) p$, where the first part is the coordinate transformation used in the method (a "point transformation") and the second part is the usual lift of a point transformation into a canonical transformation. The second or 
momentum equation can also be written $p d x=P d X$, which by integration gives

$$
s(x)=S(X),
$$

where $s$ and $S$ are the actions of the original problem and the standard problem, respectively. In fact, this equation (the equality of the actions) specifies the coordinate transformation $X=X(x)$. The geometry is illustrated in Fig. 15, in which the method of comparison equations is used to map a quantized curve of a nonlinear oscillator (the Morse oscillator, part (a) of the figure) into a quantized curve of a standard problem (the harmonic oscillator, part (b) of the figure). Equation (66) implies the equality of the shaded areas in the figure, which in turn determines the function $X(x)$. In the figure, $X_{0}=X\left(x_{0}\right)$.

The function $X(x)$ can be analytically continued to the classically forbidden regions where $p, P, s$ and $S$ all become complex, but the transformation $X=X(x)$ is real and in fact forms a single, smooth (usually analytic) coordinate transformation throughout both classically allowed and forbidden regions. See Fig. 2 of Ref. [27] for a plot of the function $X(x)$ in one example, which shows its completely smooth behavior as one passes from classically allowed to classically forbidden regions. The equation $s(x)=S(X)$ is obvious in a sense: actions are areas, and areas are preserved by canonical transformations.

We have been interested in the generalization of the method of comparison equations to a class of canonical transformations that is larger than the point transformations. Since canonical transformations are the semiclassical representatives of unitary transformations, the idea is to carry out a unitary transformation on the original problem such that the transformed problem has a standard form, to within errors of order $\hbar^{2}$, where the choice of unitary transformation is guided by geometrical criteria in the classical phase space. The standard problem is sometimes referred to as a "quantum normal form." Examples of quantum normal form calculations and an interesting perspective on Bohr-Sommerfeld or torus quantization may be found in Refs. [28, 29]. In those references only the problem of determining eigenvalues is considered, but in the present application we are interested in the transformation of the wave functions, a problem that involves extra features. As it turns out, it is not only necessary to carry out a unitary transformation (which at the classical level maps a pair of orbits into another pair that are in standard form), but also a certain nonunitary transformation (to make the densities 
of probability on the orbits come out in standard form).

In the usual method of comparison equations, the uniform approximation for the exact solution $\psi(x)$ is given by

$$
\psi(x) \approx \frac{a(x)}{A(X)} \Psi(X)
$$

where $\Psi(X)$ is the standard solution of the standard problem, and $a(x)$ and $A(X)$ are the amplitudes of the two semiclassical approximations, $a(x)$ for the original problem and $A(X)$ for the standard problem. Both amplitudes diverge at the caustics, but their ratio has a definite limit and in fact is smooth everywhere across both classically allowed and forbidden regions.

Similarly, it turns out that the uniform approximation for the $6 j$-symbol in terms of $d$-matrices is given by

$$
\left\{\begin{array}{lll}
j_{1} & j_{2} & j_{12} \\
j_{3} & j_{4} & j_{23}
\end{array}\right\} \approx(-1)^{\nu_{\mathrm{ex}}} \frac{A_{\mathrm{PR}}}{A_{d}} d_{m m^{\prime}}^{j}(\beta),
$$

where $A_{\mathrm{PR}}$ is given by (17) or (22) and $A_{d}$ by (58) or (65) in the classically allowed or forbidden regions, respectively, and where $(-1)^{\nu_{\mathrm{ex}}}$ is an extra phase defined in (74). In other words, the uniform approximation for the $6 j$ symbol is of the same form (67) that emerges from the method of comparison equations, apart from an extra phase.

\subsection{The Details of the Uniform Approximation}

At the heart of the new uniform approximation is a smooth, area-preserving map between the $6 j$-sphere and the $d$-sphere that is parameterized by fixed, quantized values of $J_{12}$ and $J_{23}$, related to quantum numbers $j_{12}$ and $j_{23}$ by (8). The area of the $6 j$-sphere and that of the $d$-sphere must be equal, which implies

$$
D=2 j+1,
$$

where $D$ is given by (7) or (12). Thus the value of the parameter $j$ of the $d$-matrix in (68) is determined.

The quantum numbers $j_{12}$ and $j_{23}$ determine a specific pair of orbits on the $6 j$-sphere, the small circle $J_{12}=j_{12}+\frac{1}{2}$, and the oval $J_{23}=j_{23}+\frac{1}{2}$. The map is required to map the small circle $J_{12}=j_{12}+\frac{1}{2}$, a quantized orbit, onto

a small circle $J_{z}=$ const on the $d$-sphere. Because area is preserved, the 
small circle on the $d$-sphere must also be quantized, and contain the same area about the north pole as the original small circle on the $6 j$-sphere. That is, we must have $j_{12, \max }-j_{12}=j-m$, or,

$$
m=j_{12}-j_{12, \text { avg }}
$$

where $j_{12, \text { avg }}=\left(j_{12, \min }+j_{12, \max }\right) / 2$. This determines the quantum number $m$ in (68). We only require our map to map this specific small circle of constant $J_{12}$ on the $6 j$-sphere onto the corresponding small circle on the $d$-sphere; other small circles of constant $J_{12}$ on the $6 j$-sphere, for other values of $J_{12}$, are not mapped to small circles of constant $J_{z}$ on the $d$-sphere.

Similarly, we require the map to map the quantized oval $J_{23}=j_{23}+\frac{1}{2}$ on the $6 j$-sphere onto a small circle on the $d$-sphere that is centered about some direction $\hat{\mathbf{n}}$ that lies on the semicircle $J_{y}=0, J_{x}>0$ on the $d$-sphere. The direction $\hat{\mathbf{n}}$ is a function of the angle $\beta$, which will be specified momentarily. Because area is preserved, the new small circle on the $d$-sphere will be a quantized orbit $J_{n}=m^{\prime}$, enclosing the same area about the axis $\hat{\mathbf{n}}$ as the oval $J_{23}=j_{23}+\frac{1}{2}$ encloses about the point $J_{23}=J_{23, \min }$. Since the minimum of $J_{23}$ corresponds to the maximum of $J_{n}$, the quantum number $m^{\prime}$ satisfies $j_{23}-j_{23, \min }=j-m^{\prime}$, or,

$$
m^{\prime}=j_{23, \text { avg }}-j_{23} .
$$

This determines the quantum number $m^{\prime}$ in (68). We only require our map to map this specific oval of constant $J_{23}$ on the $6 j$-sphere onto the corresponding small circle on the $d$-sphere; other ovals of constant $J_{23}$ on the $6 j$-sphere, for other values of $J_{23}$, are not mapped to small circles of constant $J_{z}$ on the $d$-sphere.

Finally, the parameter $\beta$ is determined by requiring that the area of the lune on the $6 j$-sphere should equal the area of the lune on the $d$-sphere. Effectively, we rotate the small circle $J_{n}=m^{\prime}$ until the two areas are equal. This is in the classically allowed region; in the classically forbidden region, the analytic continuations of the areas on the two spheres are set equal. In this way, if the $6 j$-symbol is in the classically allowed region, then so is the $d$-matrix, and vice versa. This condition is the analog of (66) in the standard method of comparison equations.

We take the classically allowed region first. We shall show elsewhere that the Ponzano-Regge phase is related to the area of the lune on the $6 j$-sphere by

$$
\Phi_{\mathrm{PR}}=\frac{1}{2}(\text { Area of lune })+\Phi_{0},
$$


where $\Phi_{0}$ is an extra phase that is related to the topology of loops in the phase space of Schwinger's oscillators, as discussed in Ref. [14. Without going into this, we can determine $\Phi_{0}$ by evaluating both the area of the lune and $\Phi_{\mathrm{PR}}$ at any point in the classically allowed region or on the caustic curve, as in Fig. 3, since $\Phi_{\mathrm{PR}}$ is a continuous function of position inside and on that curve. A point on segment A of the caustic boundary is convenient, since the area of the lune vanishes there, as shown by part A of Fig. 9. In this way we find

$$
\Phi_{0}=\left(J_{1}+J_{2}+J_{3}+J_{4}+J_{12}-J_{12, \max }\right) \pi=\left(\nu_{\mathrm{ex}}+\frac{3}{2}\right) \pi
$$

where

$$
\nu_{\mathrm{ex}}=j_{1}+j_{2}+j_{3}+j_{4}+j_{12}-j_{12 \text {,max }} .
$$

Note that $\nu_{\mathrm{ex}}$ is an integer. As a check one can evaluate $\Phi_{\mathrm{PR}}$ and the area of the lune at other points on the caustic boundary (segments BCD), and see that the answer for $\Phi_{0}$ agrees with (73).

As for the area of the lune on the $d$-sphere, it is a function of $\beta$ and is twice $\Phi_{d}$, given by (52). Altogether, the equation that must be solved for $\beta$ in the classically allowed region is

$$
\Phi_{\mathrm{PR}}=\Phi_{d}(\beta)+\Phi_{0} .
$$

In spite of the complications arising from the extra term $\Phi_{0}$, the geometrical meaning of (75) is simple: the areas of the lunes on the two spheres are equal.

Taking cosines, (75), (73) and (74) imply

$$
\cos \left(\Phi_{\mathrm{PR}}+\frac{\pi}{4}\right)=\cos \left(\Phi_{d}+\nu_{\mathrm{ex}} \pi+\frac{3 \pi}{2}+\frac{\pi}{4}\right)=(-1)^{\nu_{\mathrm{ex}}} \cos \left(\Phi_{d}-\frac{\pi}{4}\right)
$$

which explains the extra phase in (68) as well as the opposite signs on the $\pi / 4$ in the asymptotic formulas (16) and (57).

In the classically forbidden region, the analytic continuations of the areas of the two lunes become complex, but their real parts are constant in any given region $(\mathrm{ABCD})$, so only the imaginary parts need be equated. In this case, the condition is

$$
\bar{\Phi}_{\mathrm{PR}}=\bar{\Phi}_{d}(\beta),
$$

where $\bar{\Phi}_{\mathrm{PR}}$ is given by (20) and $\bar{\Phi}_{d}$ by (63) . Since both $\bar{\Phi}_{\mathrm{PR}}$ and $\bar{\Phi}_{d}$ vanish on the caustic, the condition (77) implies that if the $6 j$-symbol is on a caustic, then so is the $d$-matrix (as it should be). 
To find the root of (75) in the classically allowed region it helps to know the values of the areas of the lunes on the $d$-sphere at the two turning points $\beta_{1}$ and $\beta_{2}$, defined by (61). Call the half areas of the $d$-lunes at these two turning points $\mathcal{A}_{1}$ and $\mathcal{A}_{2}$. Then we have

$$
\mathcal{A}_{1}=\left[j+\frac{1}{2}-\max \left(m, m^{\prime}\right)\right] \pi, \quad \mathcal{A}_{2}=\max \left(0,-m-m^{\prime}\right) \pi .
$$

From these we compute an initial estimate for the root based on linear interpolation,

$$
\beta_{0}=\beta_{1}+\frac{\mathcal{A}_{1}-\Phi_{\mathrm{PR}}+\Phi_{0}}{\mathcal{A}_{1}-\mathcal{A}_{2}}\left(\beta_{2}-\beta_{1}\right),
$$

after which a Newton-Raphson iteration converges to the actual root in all the cases we have examined, without taking iterations outside the interval $\left[\beta_{1}, \beta_{2}\right]$. We have no proof that this is always true, however.

As for the classically forbidden regions, there are two of them, $0<\beta<\beta_{1}$ and $\beta_{2}<\beta<\pi$, in which we must solve (77). Here we cannot use linear interpolation to find an initial estimate of the root since $\Phi_{d}$ is not defined at $\beta=0$ or $\beta=\pi$. Instead we have simply taken

$$
\beta_{0}=\left\{\begin{array}{rr}
\frac{1}{2} \beta_{1}, \quad 0<\beta<\beta_{1}, \\
\frac{1}{2}\left(\beta_{2}+\pi\right), \quad \beta_{2}<\beta<\pi,
\end{array}\right.
$$

and then applied a Newton-Raphson iteration. Occasionally this takes us outside the given classically forbidden region, whereupon we have reset the value of $\beta$ to a point inside the region, using a simple prescription. After this, the Newton-Raphson converges to the root in all the cases we have examined. Our algorithm has proven satisfactory for our exploratory studies, but in more serious work the root finder will require more careful attention.

The Newton-Raphson method requires us to know the derivatives $d \Phi_{d} / d \beta$ or $d \bar{\Phi}_{d} / d \beta$. These also appear in the role that $\Phi_{d}$ plays as a generating function, and are equal to the momentum $p_{\beta}$ of the rigid body, of which $d_{m m^{\prime}}^{j}(\beta)$ is an eigenfunction (on the group manifold $S U(2)$ ). The derivatives are given by

$$
\frac{d \Phi_{d}}{d \beta} \quad \text { or } \quad \frac{d \bar{\Phi}_{d}}{d \beta}=-\frac{J\left|V_{d}\right|}{\sin \beta} .
$$

This applies at the intersection $\hat{\mathbf{a}}$ (not $\hat{\mathbf{a}}^{\prime}$ ) in Fig. 13, or at its analytic continuation as specified by the definitions of the angles $\bar{\alpha}_{i}$ at the end of Sec. 3.2. It is convenient in using this formula to avoid calculating the sines of $\kappa, \phi$ 
or $\eta$, which become complex in the classically forbidden region; this can be done if $\left|V_{d}\right|$ is evaluated by taking the square root of the expression in (56)).

The value of $\beta$ is smooth and well behaved as we cross from the classically allowed to forbidden regions, just as is the function $X(x)$ in the method of comparison equations. This is illustrated in Fig. 16, which uses the same parameters as Fig. 3. The heavy line in the figure is the same caustic line as in Fig. 3, and the light lines are contours of $\beta$, labeled in degrees.

Finally, we remark that the ratio of the amplitudes $A_{\mathrm{PR}} / A_{d}$ in (68) has the form $\infty / \infty$ as the caustic is approached, so a well designed numerical implementation of that formula would give special treatment to a small region around the caustic, where l'Hospital's rule would be used to avoid numerical difficulties.

\subsection{Numerical Results}

Figure 17 shows the results of numerical tests of the new uniform approximation, with comparison with the Ponzano-Regge approximation. In the figure errors are plotted as a function of $j_{12}$ for the $6 j$-symbols

$$
\left\{\begin{array}{ccc}
39 / 2 & 23 & j_{12} \\
17 / 2 & 20 & 47 / 2
\end{array}\right\} \text { and }\left\{\begin{array}{ccc}
156 & 184 & j_{12} \\
68 & 160 & 188
\end{array}\right\},
$$

in part (a) and part (b) of the figure, respectively. The values of the five fixed $j$ 's in part (b) are 8 times those in part (a). The plots show the absolute value of the difference between the exact $6 j$-symbol and the approximate value. In both parts of the figure, the curve labeled PR is the error of the Ponzano-Regge approximation, while that labeled $U$ is the error of the uniform approximation. The error of the Ponzano-Regge approximation is large near the caustics, as expected, while the error of the uniform approximation is fairly flat throughout the classically allowed region and up to the caustics. The error of both approximations falls rapidly to zero in the classically forbidden regions, as of course does the exact $6 j$-symbol.

We computed the exact $6 j$-symbol with extended precision integer arithmetic. Recently other calculations have used extended precision floating point arithmetic to study the Wigner $3 n j$-symbols and their asymptotic properties [30, 31]. Extended precision is required when summing series with alternating (or variable) sign; often the desired sum is an exponentially small residue left when much larger terms nearly cancel. With floating point arithmetic one must carry enough extra precision so that enough remains after 
the subtractions; with integer arithmetic the answer is exact, but the cancellations are still present (hence higher precision is carried on intermediate results).

In the classically allowed region the error is oscillatory, and it is possible for the Ponzano-Regge error to be less than that of the uniform approximation simply because it accidentally happens to fall near a zero of the cosine function. One such descending spike near $j_{12}=140$ can be seen in part (b) of the figure. It is clear, however, that a fair comparison of the errors in the classically allowed region must use the amplitude of the oscillatory function and ignore the oscillations. By this measure the error of the uniform approximation in Fig. [17 is approximately 30 times smaller than that of the Ponzano-Regge approximation in the center of the classically allowed region, and gets better as we approach the caustics. This ratio is nearly the same in parts (a) and (b), indicating that both errors scale in the same way with $j$. In the classically forbidden region the errors can be compared directly, without removing any oscillatory factor, and again for the values used the figure shows that the error in the uniform approximation is smaller than that in the Ponzano-Regge approximation.

The error term for the Ponzano-Regge approximation is unknown, as is that for the uniform approximation, so there is no theory by which the errors can be compared. We would expect, however, on general grounds that the two error terms should scale the same with $j$, a conclusion that is supported by the numerical evidence. That the ratio between the errors should be as small as seen in (82) and Fig. 17 was a surprise to us, and we have no explanation for it. The values of the $j$ 's chosen in that example were essentially random, but when we try other "randomly chosen" values of the $j$ 's we get similar plots. If we systematically search for $j$ values such that the error of the uniform approximation is as unfavorable as possible relative to that of the Ponzano-Regge approximation in the classically allowed region, we find cases such as

$$
\left\{\begin{array}{lll}
44 & 40 & j_{12} \\
20 & 24 & 28
\end{array}\right\},
$$

which gives the error plots in Fig. 18, In this case the two errors are comparable for an extended range of $j_{12}$. We have found no cases in which the uniform approximation is much worse than the Ponzano-Regge approximation in the classically allowed region.

There is the question of when the uniform approximation is worst in an 
absolute sense. We define the relative error as the difference between the approximation and the exact value, divided by a reference value. In the classically forbidden region, the reference value is the absolute value of the exact value. Inside the maximum part of the Airy function lobe around the turning points the reference value is the value of the Airy function, without any cosine modulation. Elsewhere in the classically allowed region we take the reference value to be the amplitude of the Ponzano-Regge approximation.

Using this definition of relative error, we have systematically searched for $j$ values that make the relative error in the uniform approximation largest. We find that they occur in cases for which $j_{12}=j_{23}=0$. This can only happen when the other four $j$ 's are equal, so we have a $6 j$-symbol of the form

$$
\left\{\begin{array}{lll}
j & j & 0 \\
j & j & 0
\end{array}\right\} .
$$

For such symbols, the relative errors in both the uniform and the PonzanoRegge approximation actually increase with $j$, reaching approximately 0.5 (uniform) or 1.1 (Ponzano-Regge) when $j=10$. The tetrahedra corresponding to $6 j$-symbols of the form (84) have two small edges $\left(j_{12}\right.$ and $j_{23}$, with lengths $J_{12}=J_{23}=\frac{1}{2}$ ) with no vertex in common, as illustrated in part (a) of Fig. 19.

The uniform approximation is asymptotic, so it is no surprise that it does not work well for small quantum numbers such as those appearing in (84). Nevertheless it is interesting to see in more detail why the approximation is not good. Figure 20 shows the $6 j$-sphere for a symbol of the form (84). The orbit $j_{12}=0\left(J_{12}=\frac{1}{2}\right)$ is the small circle about the south pole, while the orbit $j_{23}=0\left(J_{23}=\frac{1}{2}\right)$ is the curve that ends at the south pole in a cusp. The orbit $j_{23}=0$ is not smooth at the south pole, and it cannot be deformed into a small circle on the $d$-sphere by any smooth map. Since the map is not smooth, it has infinite derivatives, and the semiclassical approximation breaks down. In fact, in this case, even the $6 j$-sphere itself is not smooth. That is, the $6 j$-sphere is obtained by symplectic reduction from a higherdimensional phase space, and it is always topologically a sphere. But when the first four $j$ 's are all equal as in (84), the sphere is not differentiable at the south pole. In this case, it would be more appropriate to think of a tear drop with its cusp at the south pole, rather than a sphere.

Unlike the Ponzano-Regge approximation, the uniform approximation is not invariant under all the symmetries of the $6 j$-symbol. It is invariant under 
the three operations in which the upper and lower elements of two columns are swapped, but not under the six permutations of the columns. (We have not tested the "extra" symmetries due to Regge[2].) Therefore when $j \neq 0$, we can permute columns in an unfavorable case such as (84) to obtain a better approximation. We have tested an algorithm in which, before applying the uniform approximation, the columns of the $6 j$-symbol are permuted to place the column with the largest minimum value in the third column. Then when we search for the worst case of the uniform approximation, we find that they occur with symbols of the form

$$
\left\{\begin{array}{lll}
0 & 0 & 0 \\
j & j & j
\end{array}\right\}
$$

in which three of the $j$ 's are zero. Symbols of this form correspond to tetrahedra that look like part (b) of Fig. 19. In this case the relative error in the uniform approximation no longer grows with $j$, rather it seems to approach a limit of about .075 .

Note that if any $j$ in a $6 j$-symbol vanishes, the symbol can be evaluated trivially in closed form. Thus, no approximation is needed in the worst cases (84) and (85) that we have examined.

\section{Conclusions}

We have written computer codes that implement the uniform approximations given in this paper, which document the algorithms and check all their details. We will make these programs available to any interested parties.

We will publish the derivation of the uniform approximation presented in this paper in a future article, in which we will make explicit the symplectic map between the $6 j$-sphere and the $d$-sphere that underlies it, as well as outline how the theory of quantum normal forms leads to a uniform approximation in cases like this.

\section{Acknowledgments}

It is a pleasure to offer this manuscript as a present to Enzo Aquilanti on the occasion of his seventieth birthday, and to thank him for suggesting the problem of uniform approximations to the $6 j$-symbol. We would also like to 
thank him for years of friendship and intellectual stimulation, which we hope and trust is only a beginning.

\section{A Constructing the tetrahedron}

The $6 j$-symbol specifies the lengths $J_{i}$ of the classical angular momentum vectors $\mathbf{J}_{i}$ but not their directions, so there is the question of how the actual vectors can be constructed in three dimensional space, satisfying the identities (2) and (3).

Initially we assume that real vectors $\mathbf{J}_{i}$ exist, and we define

$$
\begin{aligned}
& \mathbf{A}_{1}=\mathbf{J}_{1} \\
& \mathbf{A}_{2}=\mathbf{J}_{1}+\mathbf{J}_{2}=\mathbf{J}_{12}, \\
& \mathbf{A}_{3}=\mathbf{J}_{1}+\mathbf{J}_{2}+\mathbf{J}_{3}=-\mathbf{J}_{4},
\end{aligned}
$$

which are the three vectors running along the edges emanating from the upper vertex in Fig. 2. We arrange these vectors as columns of a $3 \times 3$ matrix $F$, and we let $G=F^{T} F$, where $T$ means transpose. Then $G$ is the symmetric, nonnegative definite Gram matrix of dot products,

$$
G_{i j}=\mathbf{A}_{i} \cdot \mathbf{A}_{j}
$$

By using the geometry of the three triangles spanned by the $\mathbf{A}_{i}$, the components of $G$ can be found in terms of the lengths $J_{i}$,

$$
\begin{gathered}
G_{11}=A_{1}^{2}=J_{1}^{2}, \quad G_{22}=A_{2}^{2}=J_{12}^{2}, \quad G_{33}=A_{3}^{2}=J_{4}^{2}, \\
G_{12}=G_{21}=\mathbf{A}_{1} \cdot \mathbf{A}_{2}=\frac{1}{2}\left(J_{12}^{2}+J_{1}^{2}-J_{2}^{2}\right), \\
G_{13}=G_{31}=\mathbf{A}_{1} \cdot \mathbf{A}_{3}=\frac{1}{2}\left(J_{1}^{2}+J_{4}^{2}-J_{23}^{2}\right), \\
G_{23}=G_{32}=\mathbf{A}_{2} \cdot \mathbf{A}_{3}=\frac{1}{2}\left(J_{12}^{2}+J_{4}^{2}-J_{3}^{2}\right) .
\end{gathered}
$$

Alternatively, without making any assumptions about the existence of the $\mathbf{J}_{i}$, we can define $G$ in terms of the lengths $J_{i}$ by (88). Then there is the question of whether vectors $\mathbf{A}_{i}$ exist such that (87) is satisfied.

The diagonalization of $G$ is closely related to the singular value decomposition of $F$. The latter is $F=U D V^{T}$, where $U$ and $V$ are real orthogonal matrices and $D$ is a real diagonal matrix, containing the real singular values $d_{i}$ on the diagonal. But this implies $G=V D^{2} V^{T}$, so $V$ is the orthogonal

matrix that diagonalizes $G$ and the eigenvalues of $G$ are $d_{i}^{2}$. Therefore to 
find $F$ we first construct $G$ by (88) and diagonalize it, obtaining $V$ and the eigenvalues of $G$. If these eigenvalues are all nonnegative, then their square roots are the singular values, and the matrix $D$ is determined. This does not determine $U$, but that matrix amounts to an overall rotation of the tetrahedron which is arbitrary anyway. So we can set $U$ to anything convenient, such as the identity. Then we have $F=D V^{T}$, and the vectors $\mathbf{A}_{i}$ can be obtained as the columns of $F$. From these we can find the J's by inverting (6ㅜ) and using (3).

Thus a real tetrahedron can be constructed if and only if the eigenvalues of $G$ are nonnegative. The tetrahedron is only determined modulo overall rotations, proper and improper. If we wish the tetrahedron to have a definite handedness, we can perform a spatial inversion, if necessary, to make the volume in (13) positive. The spatial inversion is properly brought about by time reversal, not parity, which does not change the sign of angular momenta. With this understanding, the final tetrahedron is determined modulo proper rotations.

If any of the eigenvalues of $G$ are negative, then a real tetrahedron does not exist. It turns out that at most one eigenvalue of $G$ defined by (88) can be negative, assuming the triangle inequalities on the $J_{i}$. A proof of this fact is easily given by considering the secular polynomial of $G$, whose coefficients can be expressed in terms of the lengths of the $\mathbf{J}$ 's and the areas of the triangles formed by them. If $G$ has one negative eigenvalue, then we can order the singular values so that the imaginary one is the third one. Then from $F=D V^{T}$ we see that the tetrahedron can be constructed with complex vectors, in which the $x$ - and $y$-components of the J's are real, and the $z$-components are purely imaginary.

\section{References}

[1] Edmonds A R 1960 Angular Momentum in Quantum Mechanics (Princeton: Princeton University Press)

[2] Biedenharn L C and Louck J D 1981 The Racah-Wigner Algebra in Quantum Theory (Reading, Massachusetts: Addison-Wesley)

[3] Brink D M and Satchler G R 1993 Angular Momentum (Oxford: Oxford University Press) 
[4] Varshalovich D A, Moskalev A N and Khersonskii V K 1981 Quantum Theory of Angular Momentum (Singapore: World Scientific)

[5] De Fazio D, Cavalli S and Aquilanti V 2003 Int. J. Quantum Chem. 93 91

[6] Anderson R W and Aquilanti V 2006 J. Chem. Phys. 24214104

[7] Aquilanti V, Cavalli S and De Fazio D 1995 J. Phys. Chem. 9915694

[8] Aquilanti V, Cavalli S and Coletti C 2001 Chem. Phys. Lett. 344587

[9] Aquilanti V and Coletti C 2001 Chem. Phys. Lett. 344601

[10] Ponzano G and Regge T 1968 in Spectroscopy and Group Theoretical Methods in Physics ed F Bloch et al (Amsterdam: North-Holland) p 1

[11] Schulten K and Gordon R G 1975 J. Math. Phys. 16 1961, ibid 161971

[12] Roberts J 1999 Geometry and Topology 321

[13] Charles L 2008 On the quantization of polygon spaces Preprint math 0806.1585

[14] Aquilanti V, Haggard H M, Littlejohn R G and Yu Liang 2007 J Phys A 405637

[15] Braun P A 1993 Rev Mod Phys 65115

[16] Kapovich M and Millson J J 1996 J Differential Geometry 44479

[17] Abraham R and Marsden J E 1978 Foundations of Mechanics (Reading, Massachusetts: Benjamin/Cummings)

[18] Biedenharn L C and van Dam H 1965 Quantum Theory of Angular Momentum (New York: Academic Press)

[19] Miller W H 1974 Adv Chem Phys 2569

[20] Goldstein H 1980 Classical Mechanics 2nd ed (Reading, Massachusetts: Addison-Wesley)

[21] Wigner E P 1959 Group Theory (New York: Academic Press) 
[22] Littlejohn R G 1990 J. Math. Phys. 312952

[23] Brussaard P J and Tolhoek H A 1957 Physica 23955

[24] Braun P A, Gerwinski P, Haake F and Schomerus H 1996 Z Phys B 100 115

[25] Sokolovski D and Connor J N L 1999 Chem Phys Lett 305238

[26] Berry M V and Mount K E 1972 Rep Prog Phys 35315

[27] Cargo M C and Littlejohn R G 2002 Phys Rev E 65026703

[28] Cargo M, Gracia-Saz A, Littlejohn R G, Reinsch M and de M Rios P 2005 J Phys A 381977

[29] Cargo M, Gracia-Saz and Littlejohn R G 2006 Multidimensional quantum normal forms Preprint math-ph 0507032

[30] Anderson R W, Aquilanti V and Ferreira C da S 2008 J. Chem. Phys. 129161101

[31] Ragni M, Bitencourt A C P, Ferreira C da S, Aquilanti V, Anderson R W and Littlejohn R G 2009 Int. J. Quantum Chem. in press. 
Figure 1: The $6 j$-symbol as a function of $j_{12}$ for $j_{1}=16, j_{2}=80, j_{3}=208$, $j_{4}=272$ and $j_{23}=276$. Sticks are the values of the $6 j$-symbol, and the curve is the Ponzano-Regge approximation.

Figure 2: A tetrahedron of positive volume with conventional labeling of edges by angular momentum vectors.

Figure 3: The $J_{12}-J_{23}$ plane for $j_{1}=\frac{9}{2}, j_{2}=3, j_{3}=\frac{11}{2}, j_{4}=6$. The classical bounds are $J_{12, \min }=\frac{3}{2}, J_{12, \max }=\frac{17}{2}, J_{23, \min }=\frac{5}{2}, J_{23, \max }=\frac{19}{2}$. The dimension of the matrix $\left\langle j_{12} \mid j_{23}\right\rangle$ is $D=\operatorname{dim} Z=7$. The point $J_{12}=5$, $J_{23}=9\left(j_{12}=\frac{9}{2}, j_{23}=\frac{17}{2}\right)$ is very close to the caustic line, but lies just inside. The Ponzano-Regge approximation is too large by a factor of 7 at this point.

Figure 4: A sequence of four flat tetrahedra, moving around the caustic line of Fig. 3 in a clockwise direction from point Y. The parameters are the same as in Fig. 3. The numbers 1, 2 etc refer to vectors $\mathbf{J}_{1}, \mathbf{J}_{2}$, etc.

Figure 5: Definition of the interior dihedral angle $\phi_{12}$. The other interior dihedral angle $\phi_{23}$ is defined similarly.

Figure 6: The phase space of the $6 j$-symbol is a sphere of radius $D / 2$ in a space in which $\left(K_{x}, K_{y}, K_{z}\right)$ are Cartesian coordinates. To within an additive constant, $K_{z}$ is $J_{12}$ and the azimuthal angle $\phi_{12}$ is the dihedral angle of the tetrahedron. Several curves of constant $J_{12}$ (small circles) are shown.

Figure 7: Curves of constant $J_{23}$ on the $6 j$-sphere. The first view shows the north pole and the point $J_{23}=J_{23 \text {,min }}$, and the second shows the south pole and the point $J_{23}=J_{23, \max }$.

Figure 8: In part (a), the classically allowed region, an orbit of constant $J_{12}$ intersects an orbits of constant $J_{23}$. The shaded area is the Ponzano-Regge phase, to within an additive constant. In part (b), the classically forbidden region, the orbits do not intersect. 
Figure 9: Caustics occur when the curve $J_{12}=$ const is tangent to the curve $J_{23}=$ const. The four types of such tangencies are illustrated.

Figure 10: Definition of Euler angle $\beta$ and unit vector $\hat{\mathbf{n}}$.

Figure 11: Curves of constant $J_{z}$ and $J_{n}$ may intersect in the classically allowed region (a), or not intersect in the classically forbidden region (b).

Figure 12: The square identifies the bounds on the classical observables $J_{z}$ and $J_{n}$, while the spots indicate the quantized values $J_{z}=m, J_{n}=m^{\prime}$. The ellipse is the caustic curve.

Figure 13: Vector â points to the intersection of the $J_{z}$-orbit with the $J_{n^{-}}$ orbit, with $J_{y}>0$. Vectors $\hat{\mathbf{z}}$, $\hat{\mathbf{n}}$ and $\hat{\mathbf{a}}$ define a spherical triangle, with interior angles $\phi, \eta$ and $\kappa$.

Figure 14: Caustics of the $d$-matrices occur when the two small circles $J_{z}=$ const and $J_{n}=$ const are tangent. There are four possible configurations.

Figure 15: In the method of comparison equations, the phase space of a nonlinear oscillator (a) is mapped into the phase space of the harmonic oscillator (b). The function $X(x)$ is determined by the equality of areas; for example, in the figure the shaded areas are equal, and $X_{0}=X\left(x_{0}\right)$.

Figure 16: Contours of $\beta$, the root of (75) or (77), in the $J_{12^{-}} J_{23}$ plane. Parameters are same as in Fig. 3 , but quantized values are omitted.

Figure 17: Absolute value of the error of the Ponzano-Regge approximation $(\mathrm{PR})$ and of the uniform approximation (U) as a function of $j_{12}$ for values of the other five $j$ 's shown in (82). The error is defined as the difference between the approximate value and the exact value.

Figure 18: Comparison of Ponzano-Regge (PR) and uniform (U) errors as a function of $j_{12}$ for the $6 j$-symbol (83). Dotted curve is the error in the uniform approximation. 
Figure 19: The uniform approximation is worst for tetrahedra that look like part (a), where the small edges are $j_{12}$ and $j_{23}$. If columns are permuted to optimize the approximation, the worst case involves tetrahedra that look like part (b), where three edges are small.

Figure 20: The orbits $j_{12}=0$ is a small circle about the south pole, while the orbit $j_{23}=0$ has a cusp at the south pole. The shaded area is the "lune." 


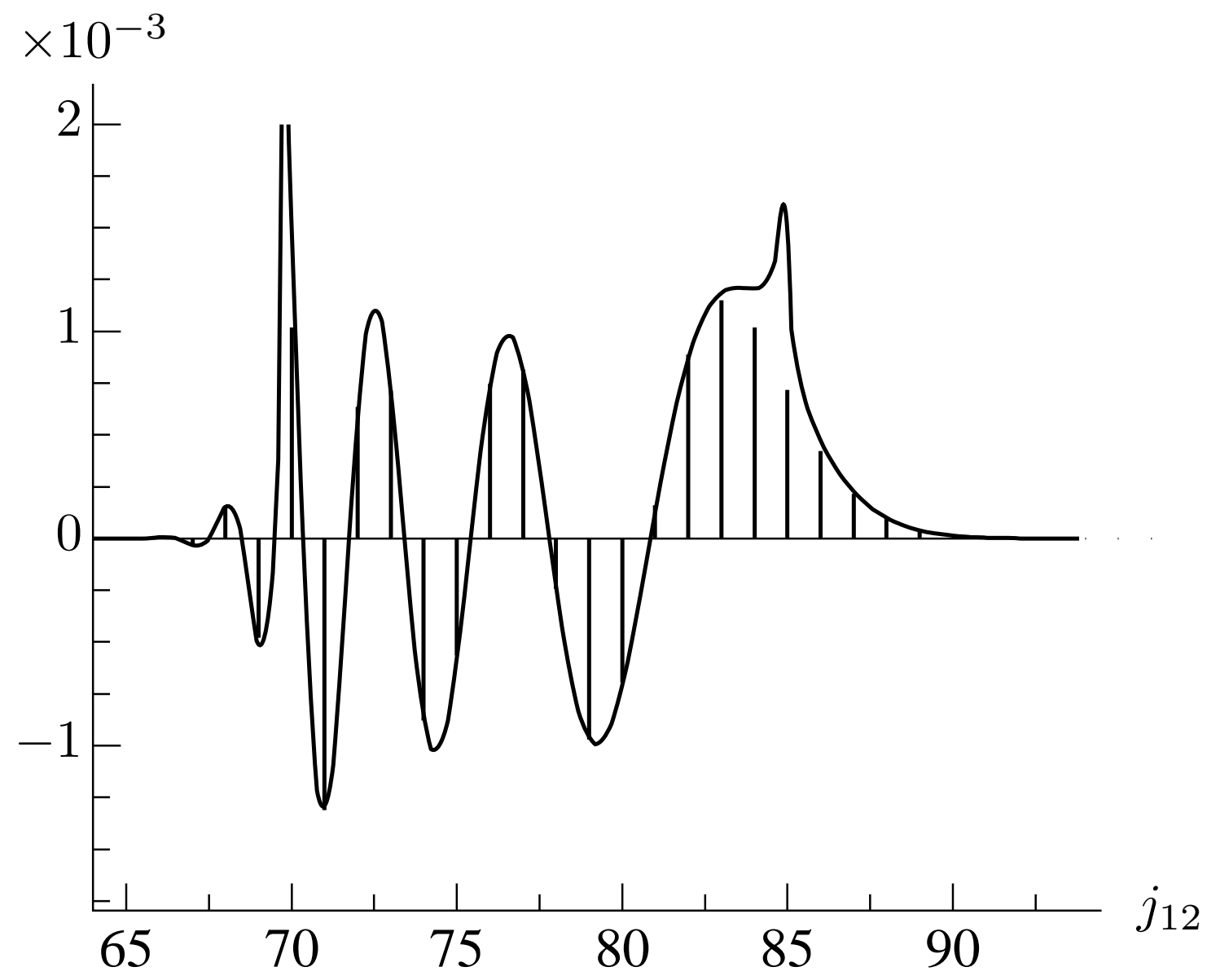




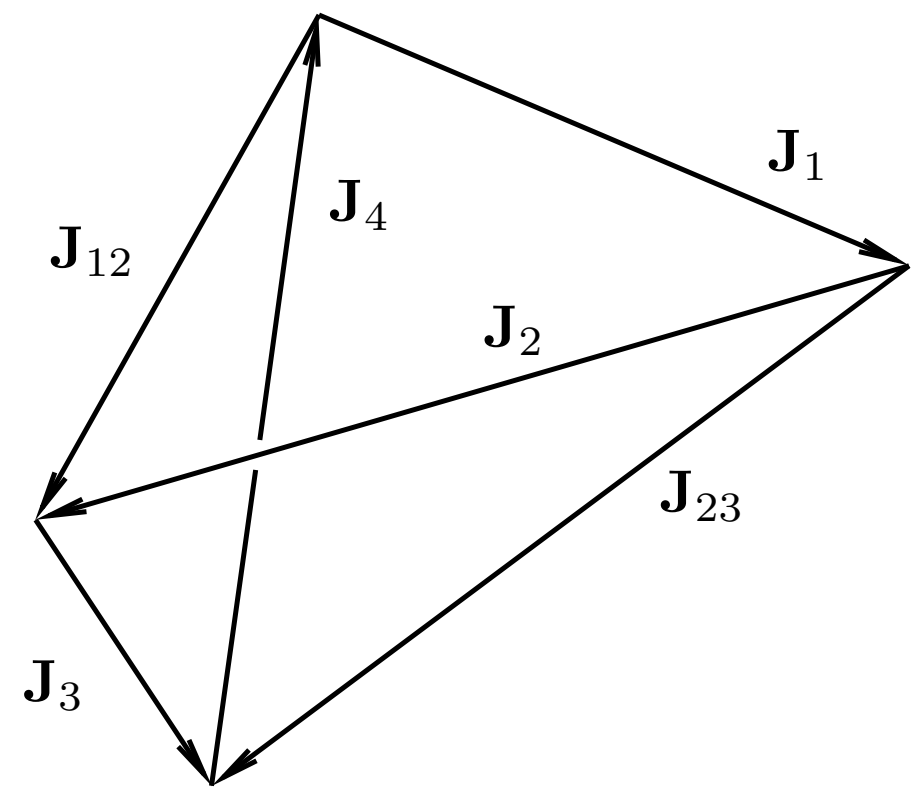




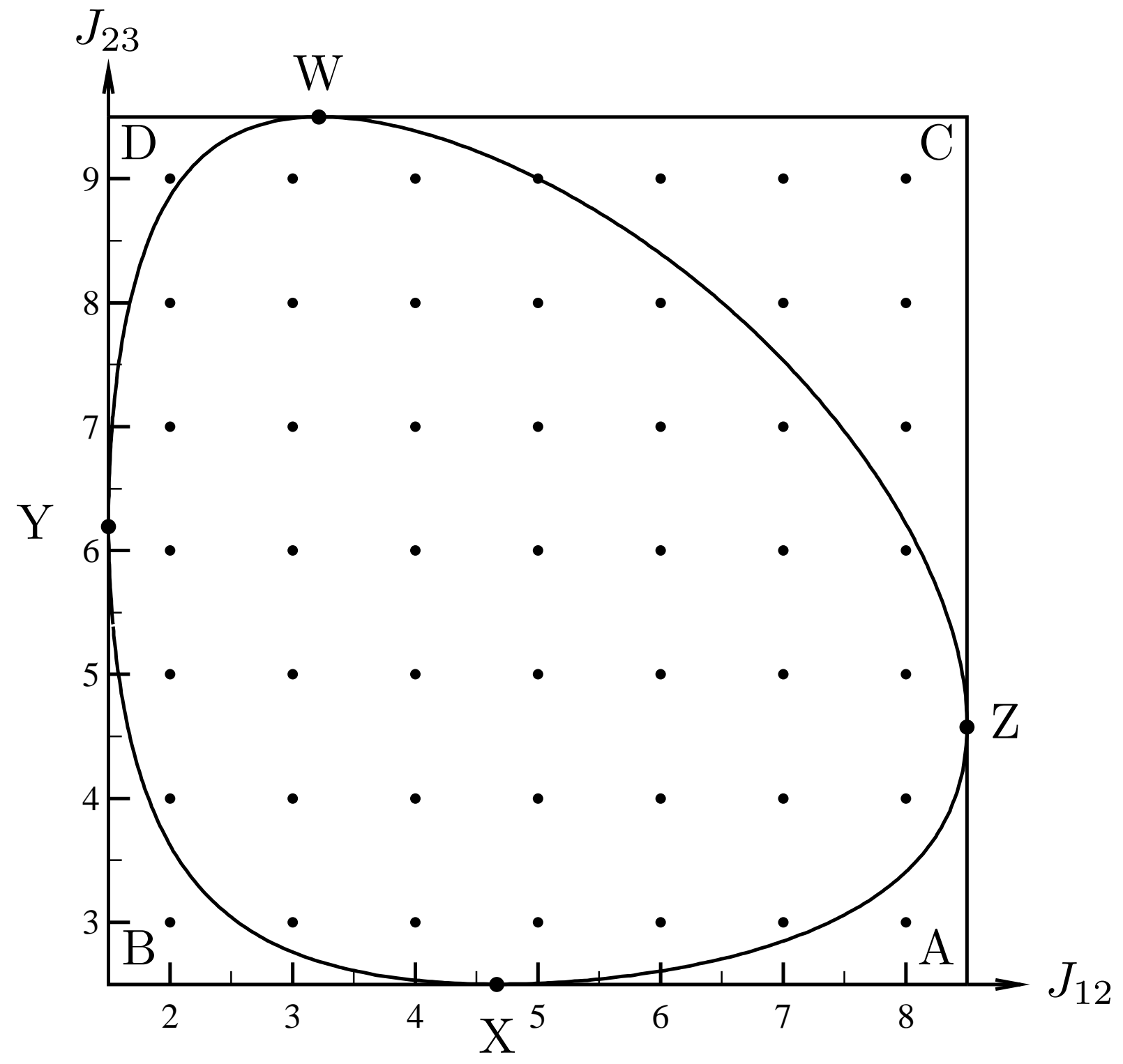



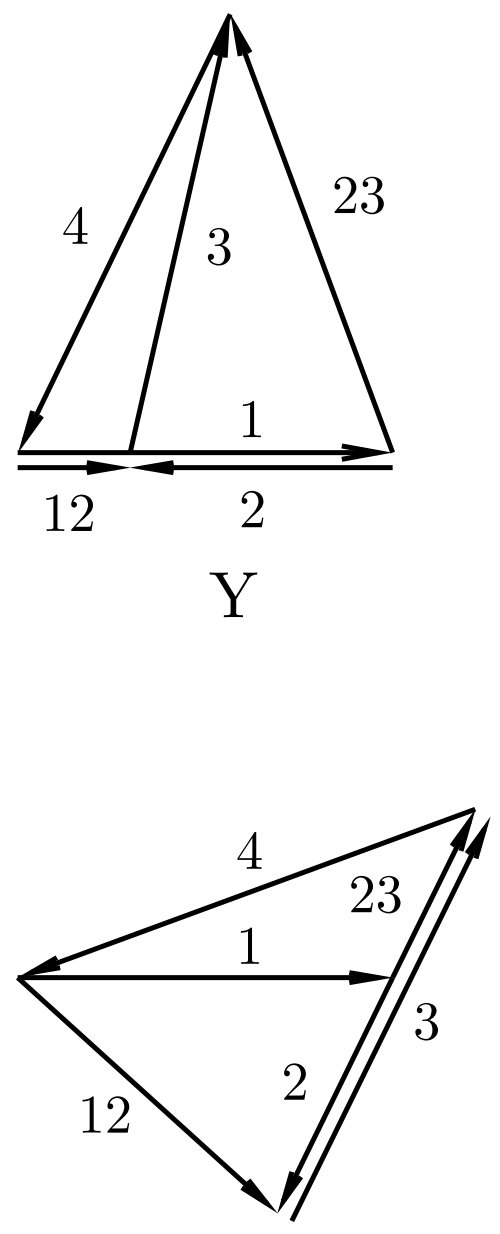

$\mathrm{X}$

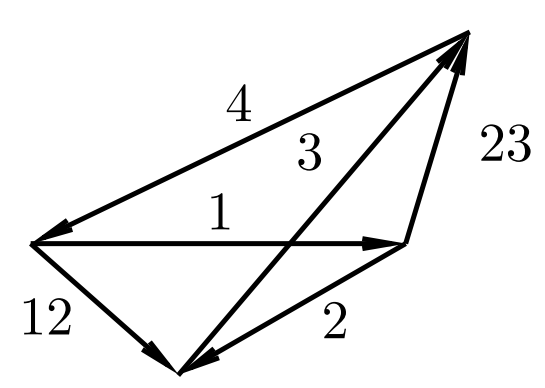

$\mathrm{Y} \rightarrow \mathrm{X}$

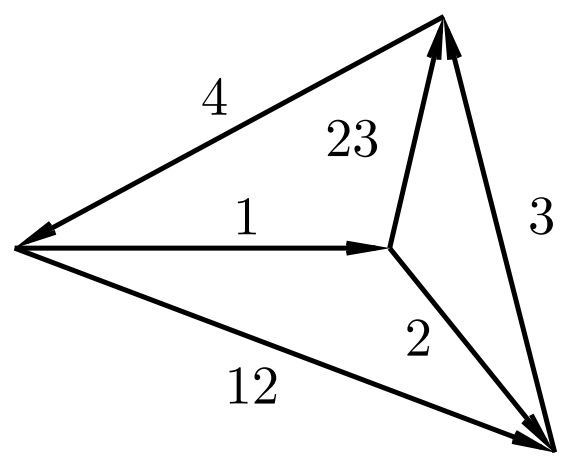

$X \rightarrow Z$ 


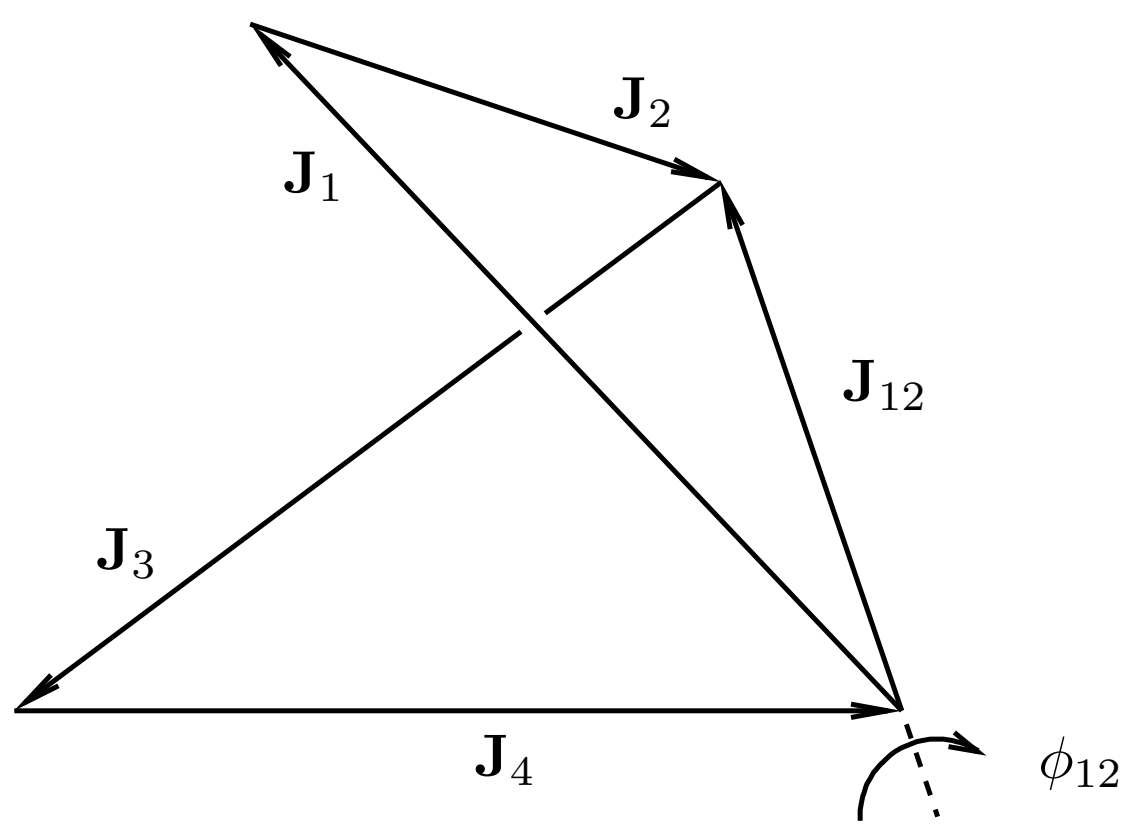




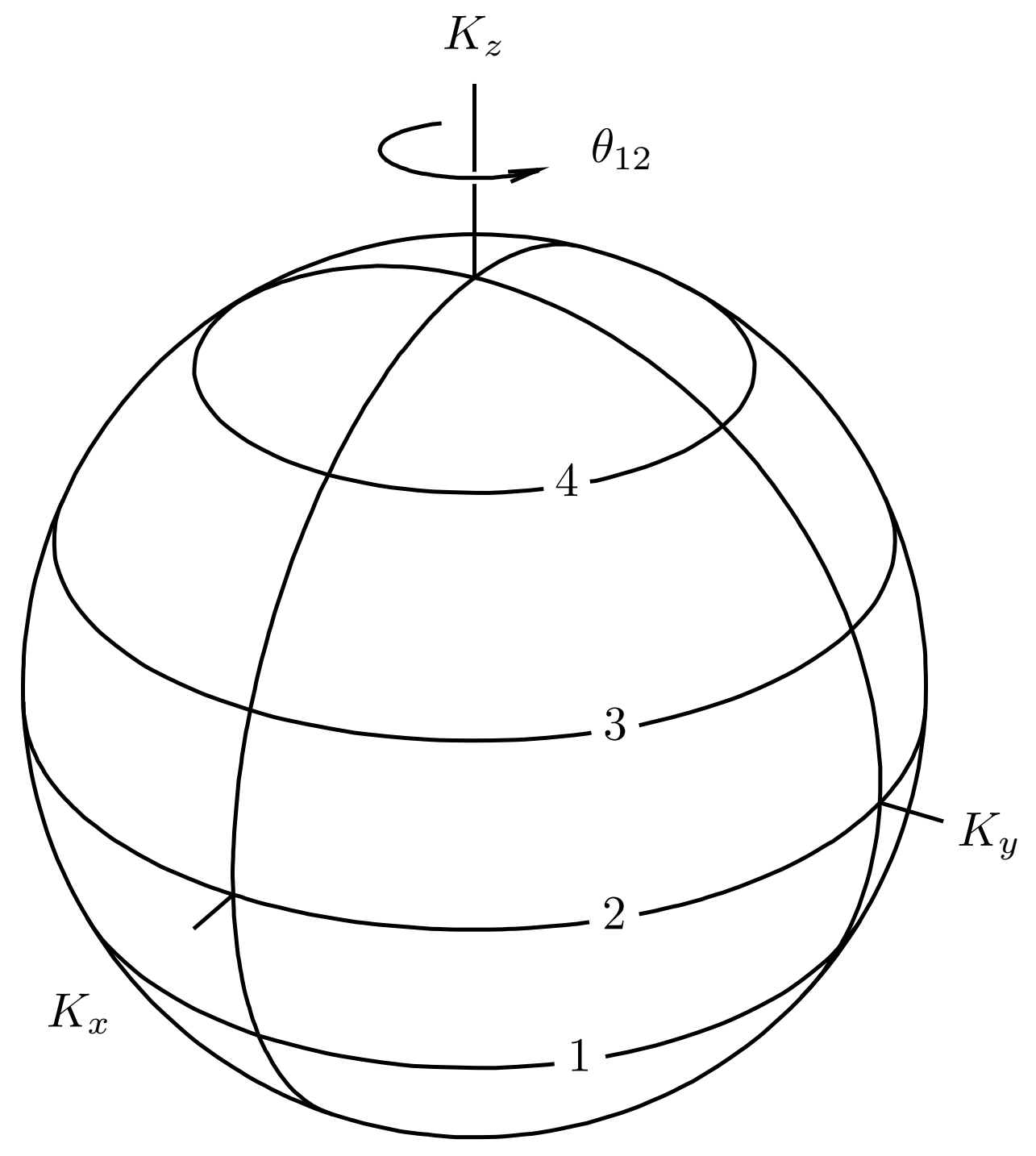



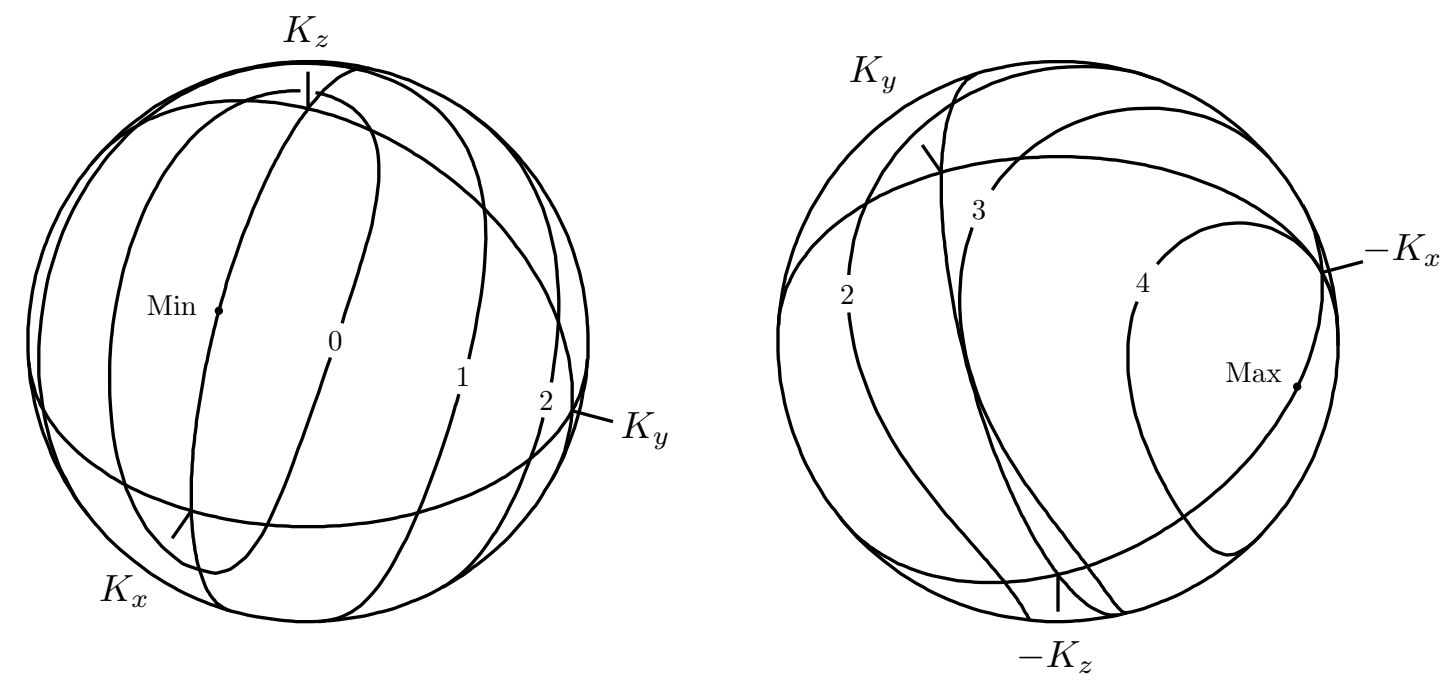


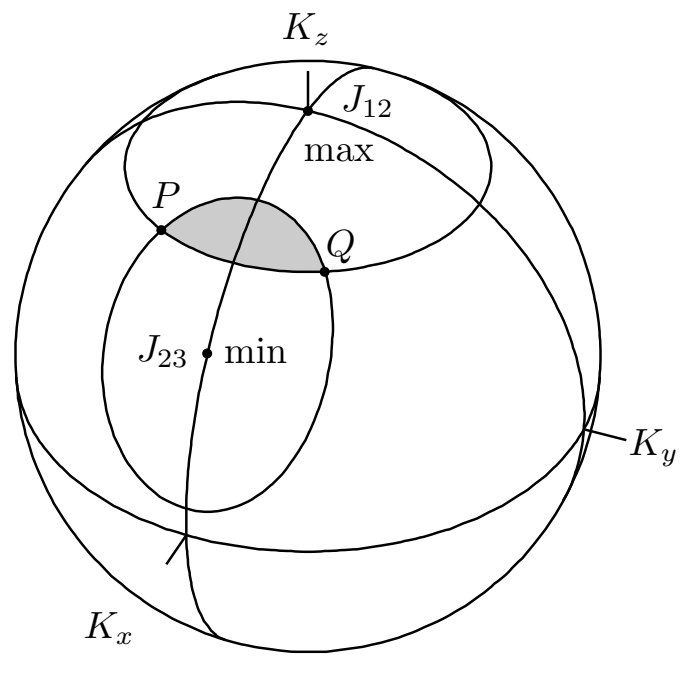

(a)

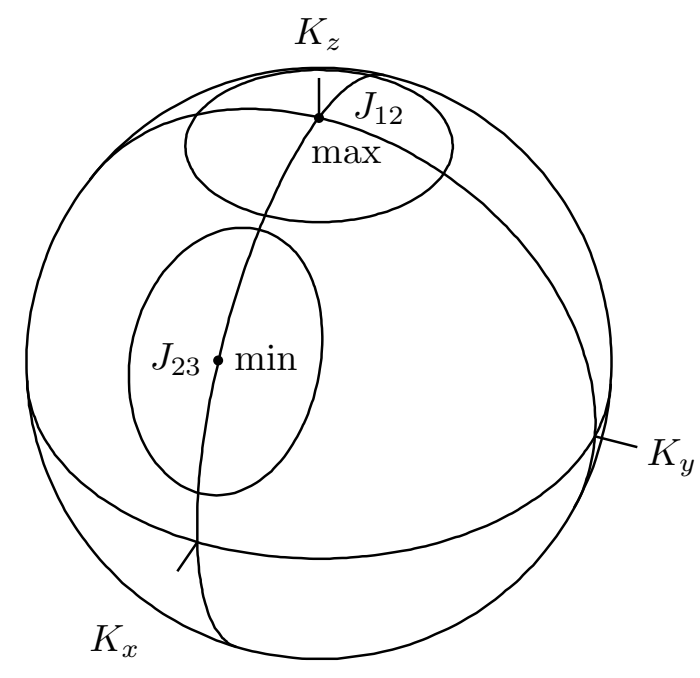

(b) 

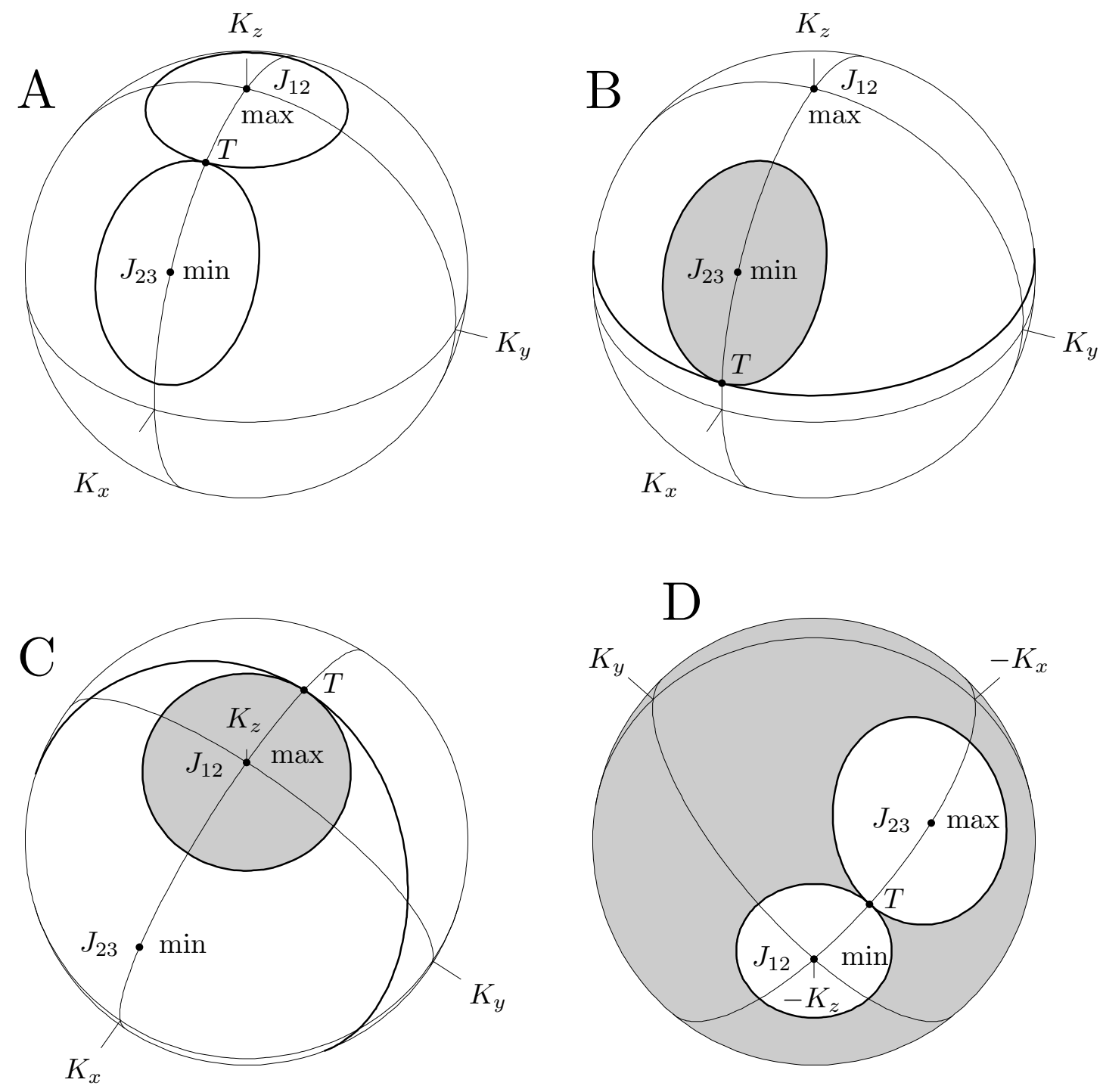


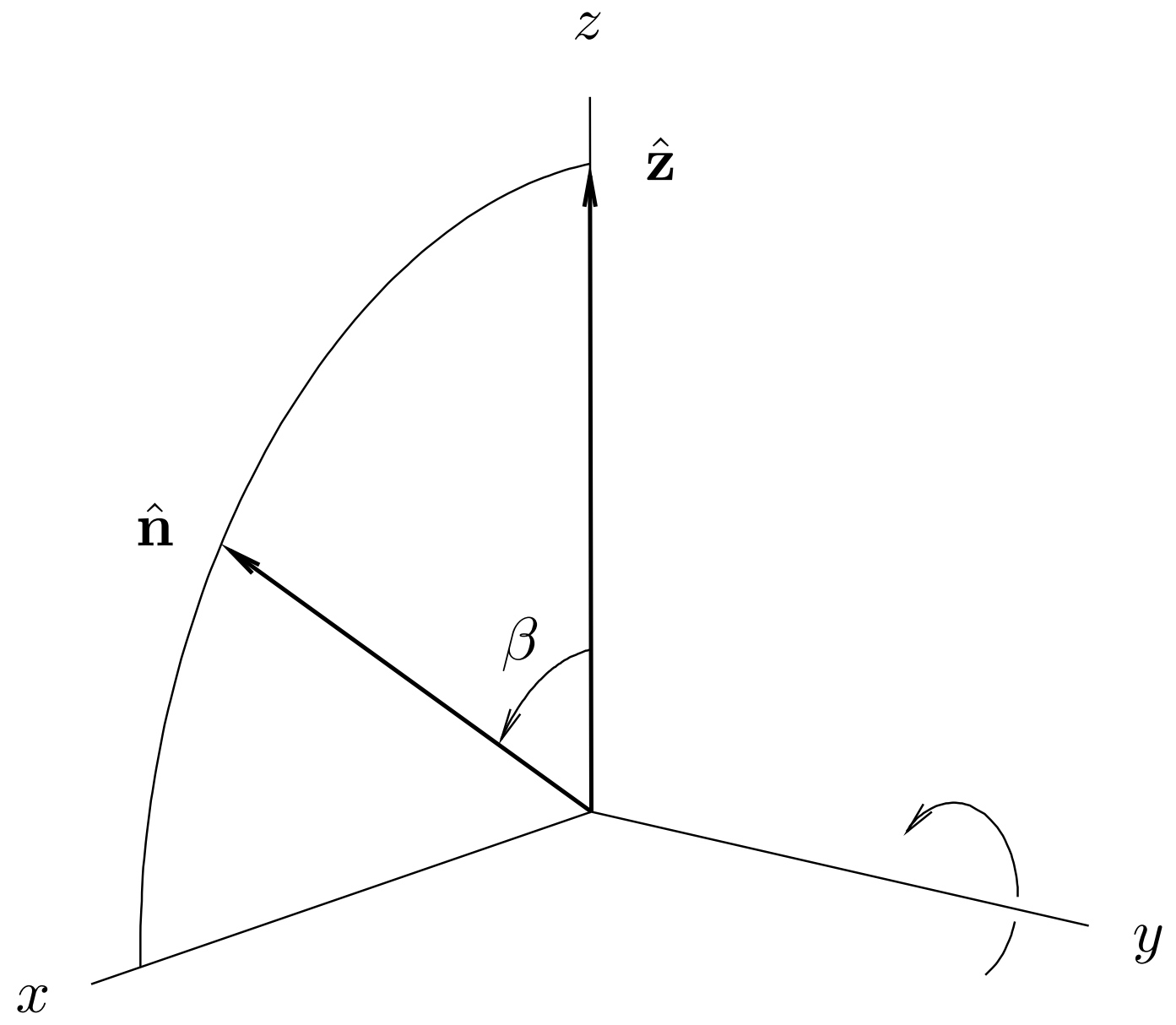



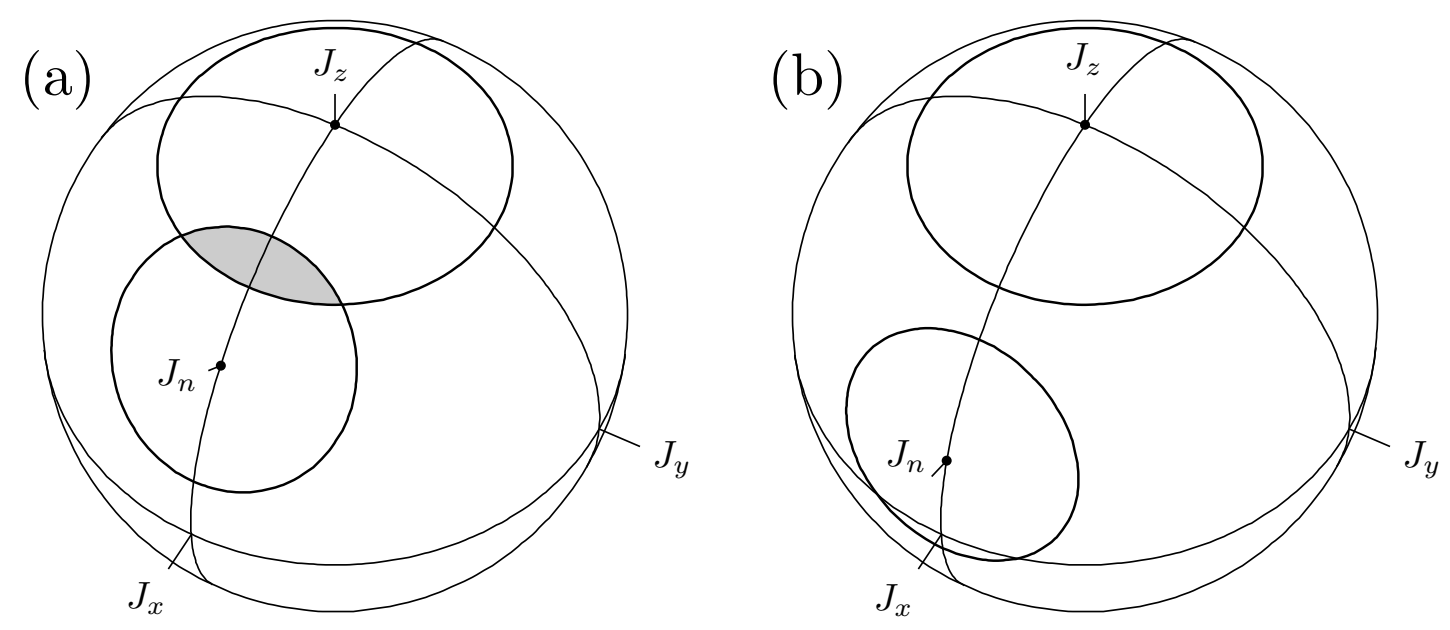


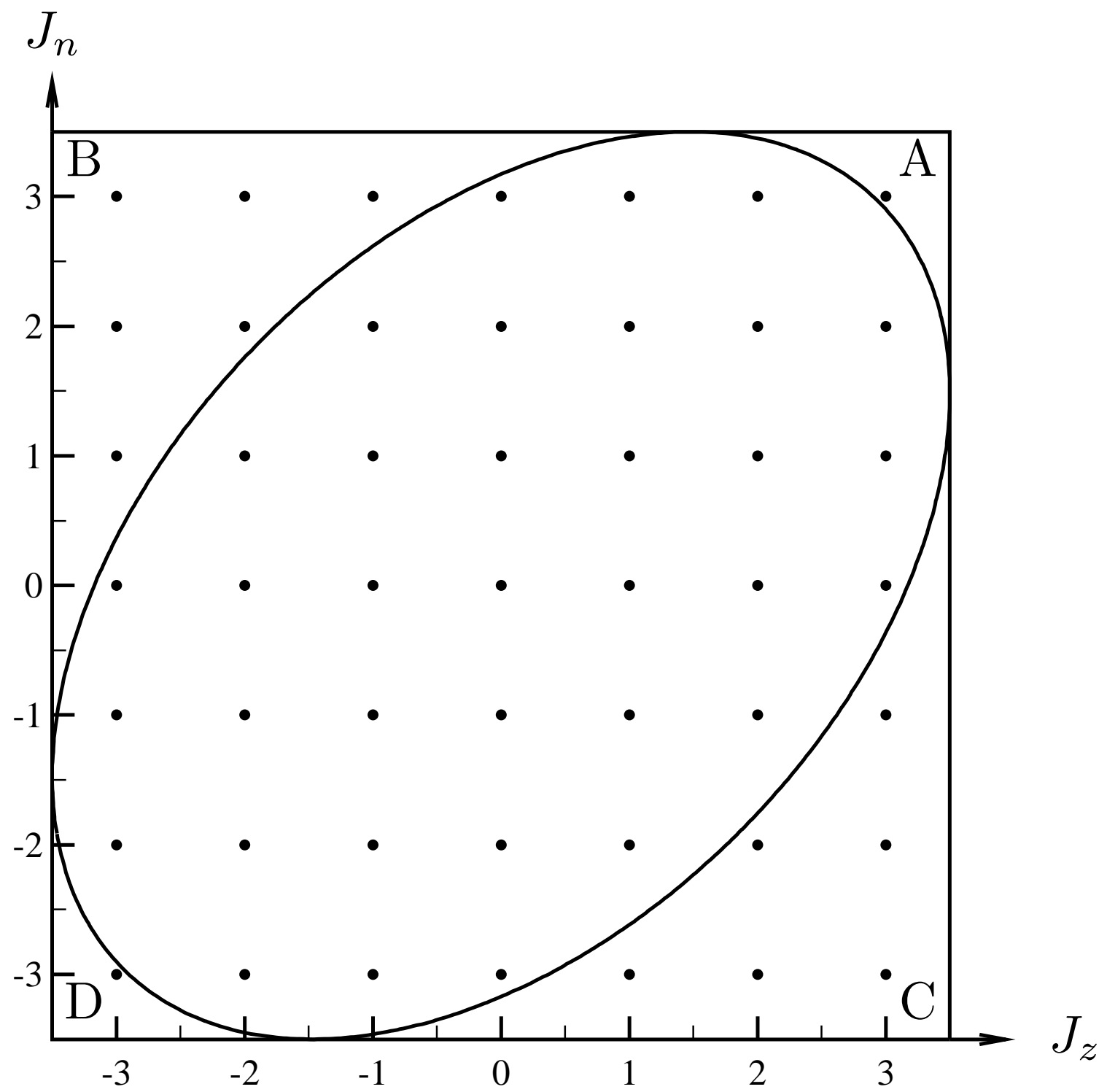




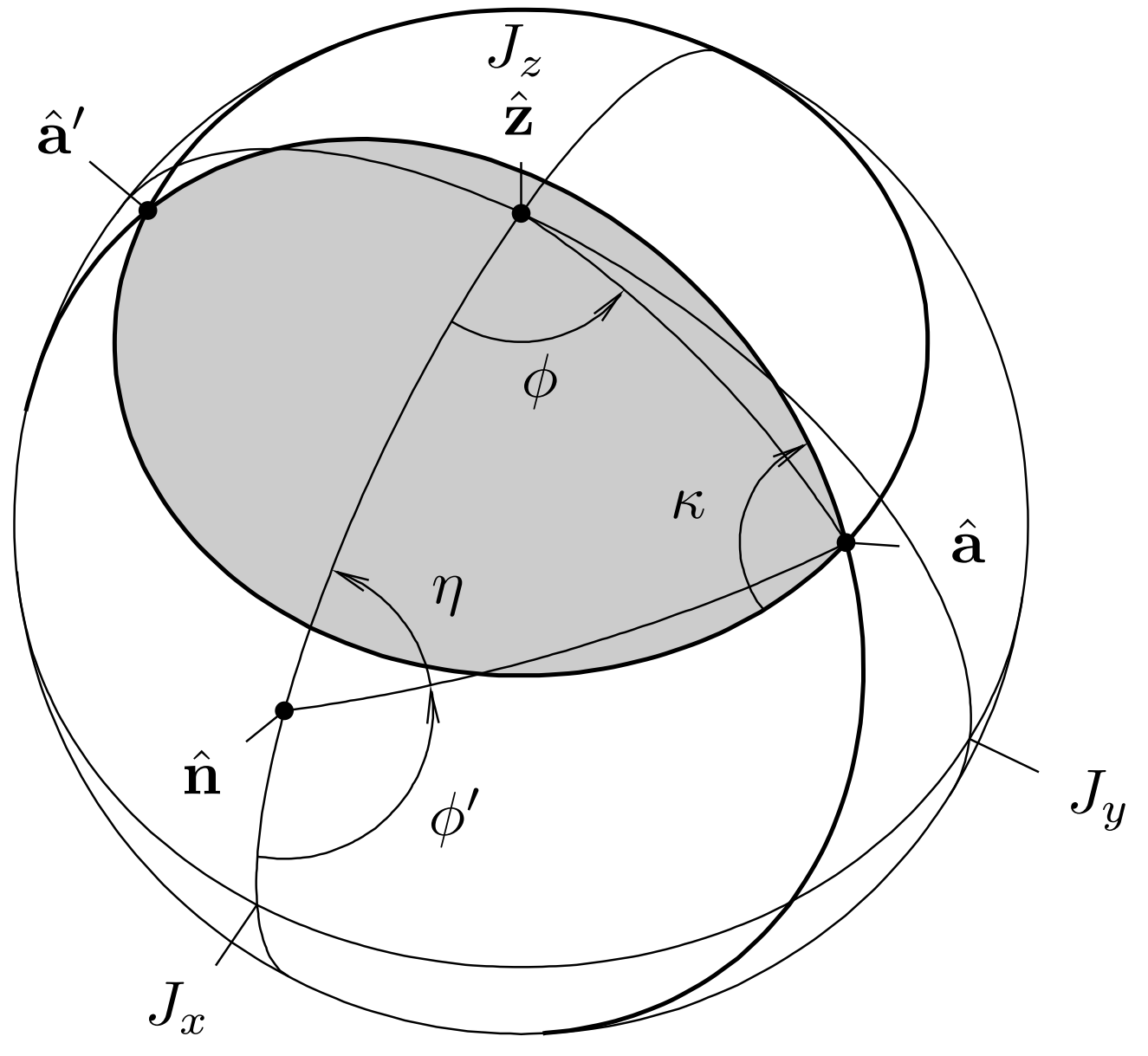



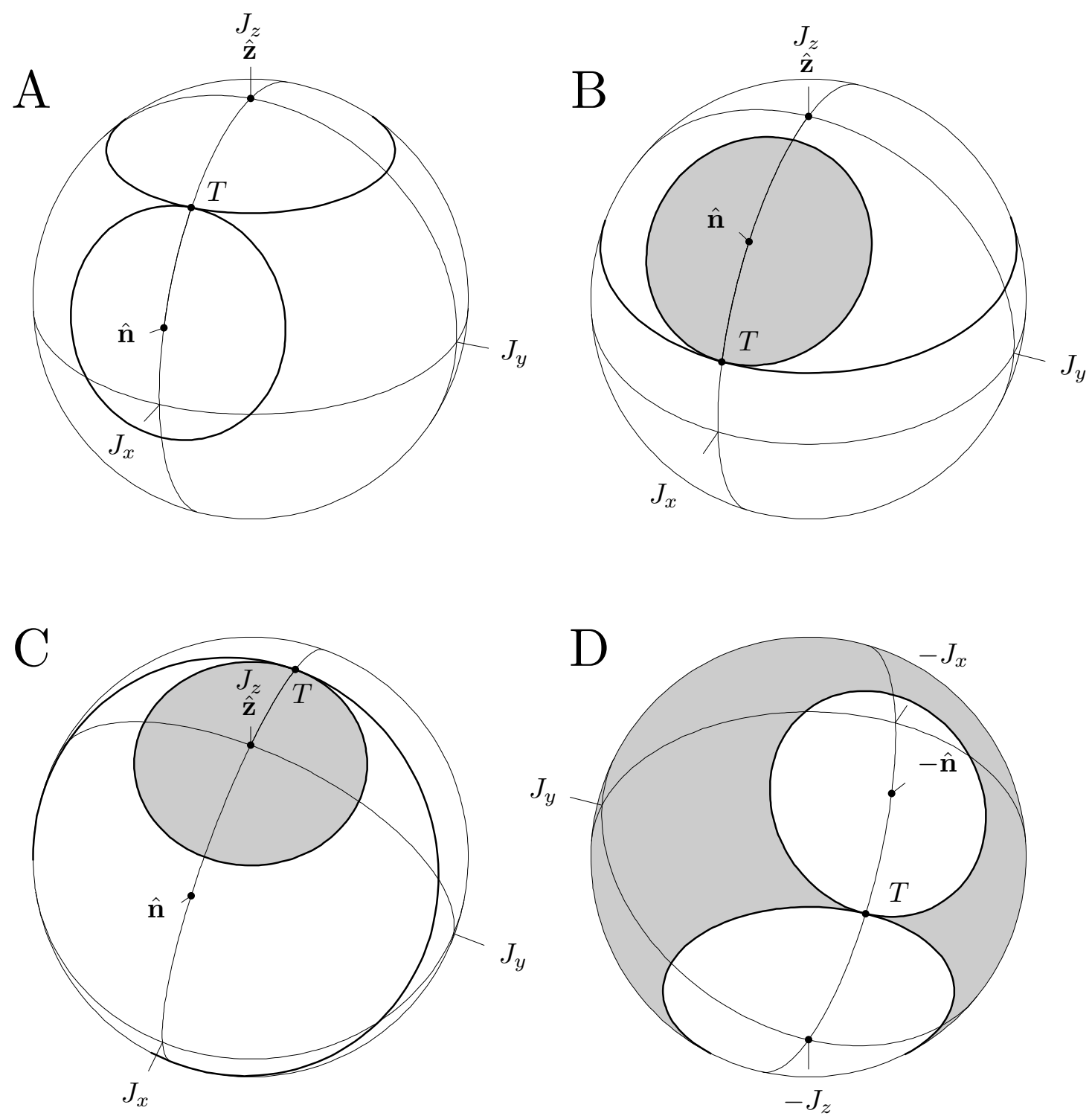

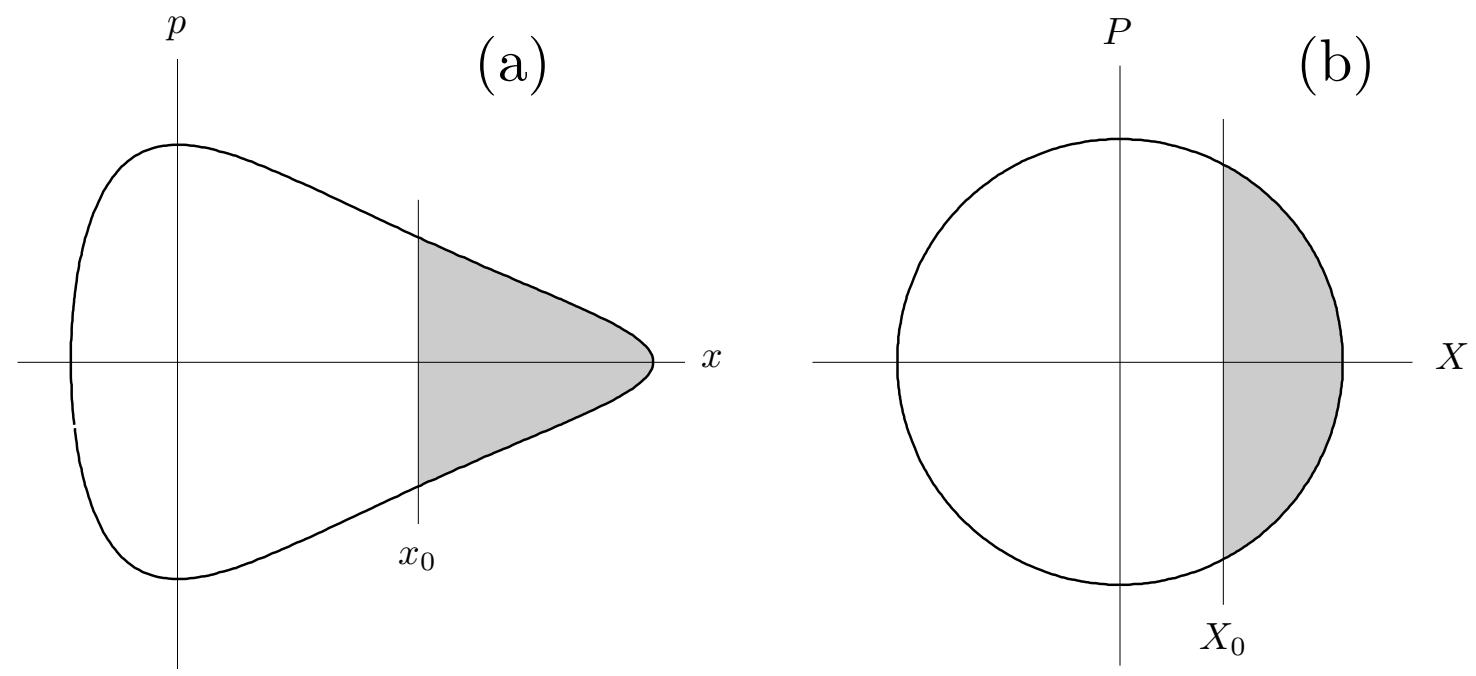


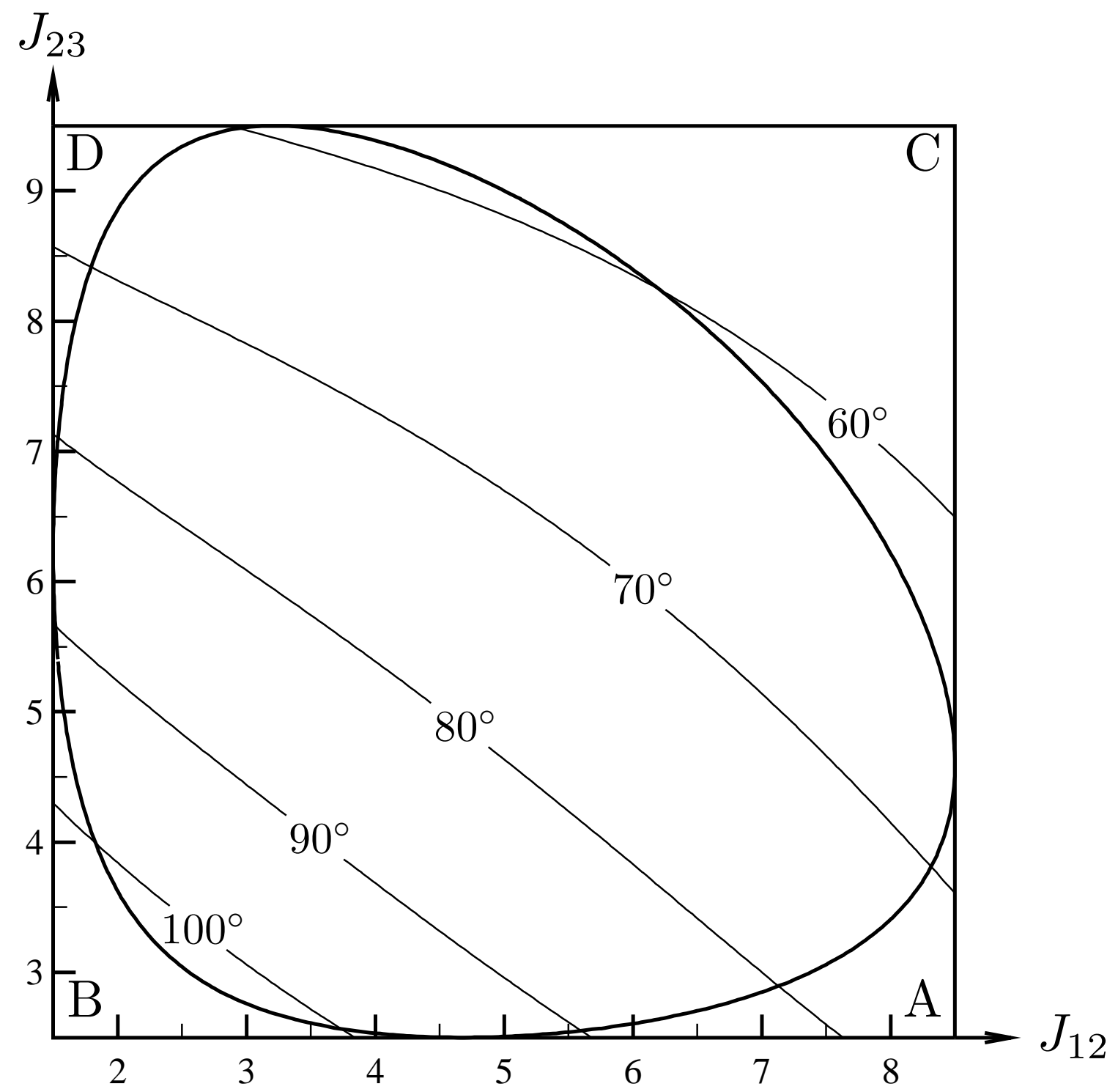



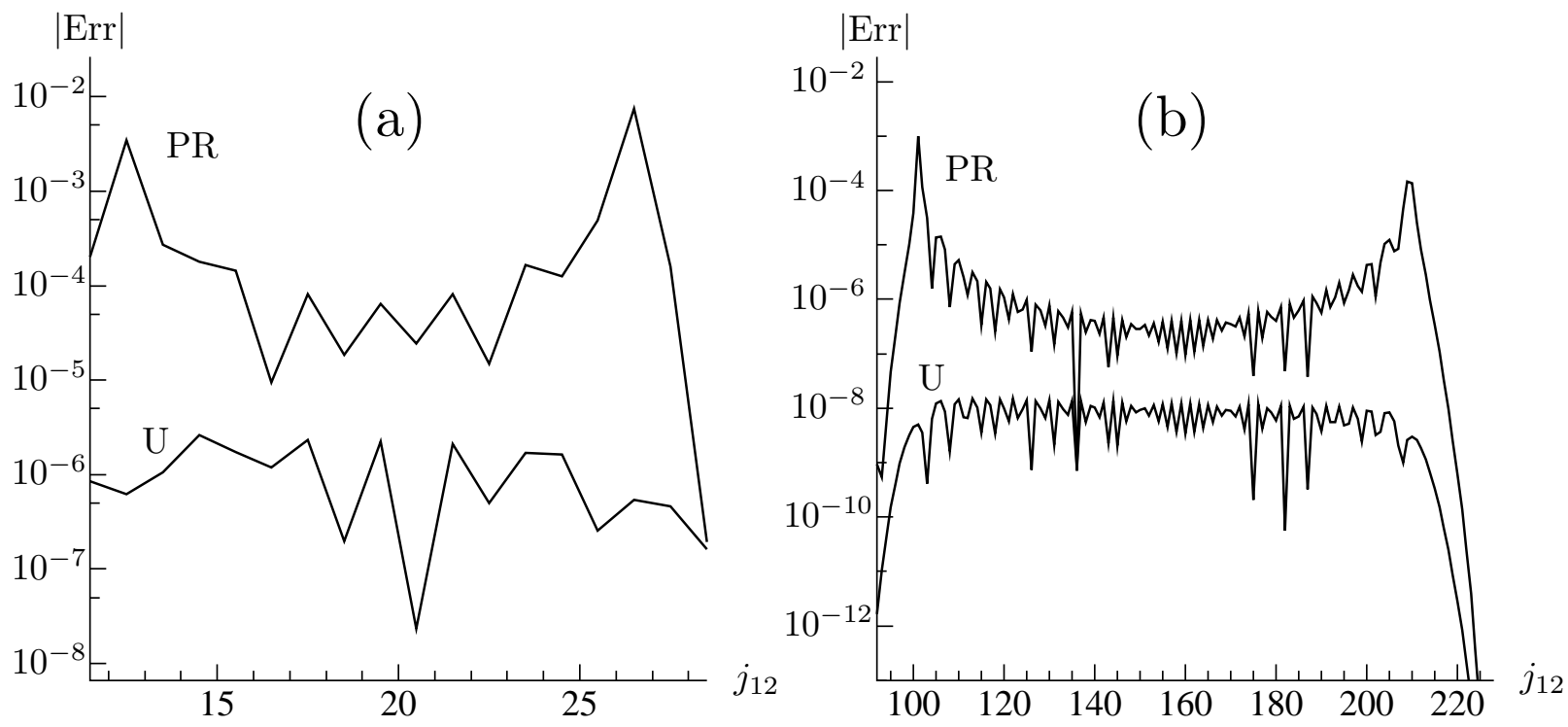


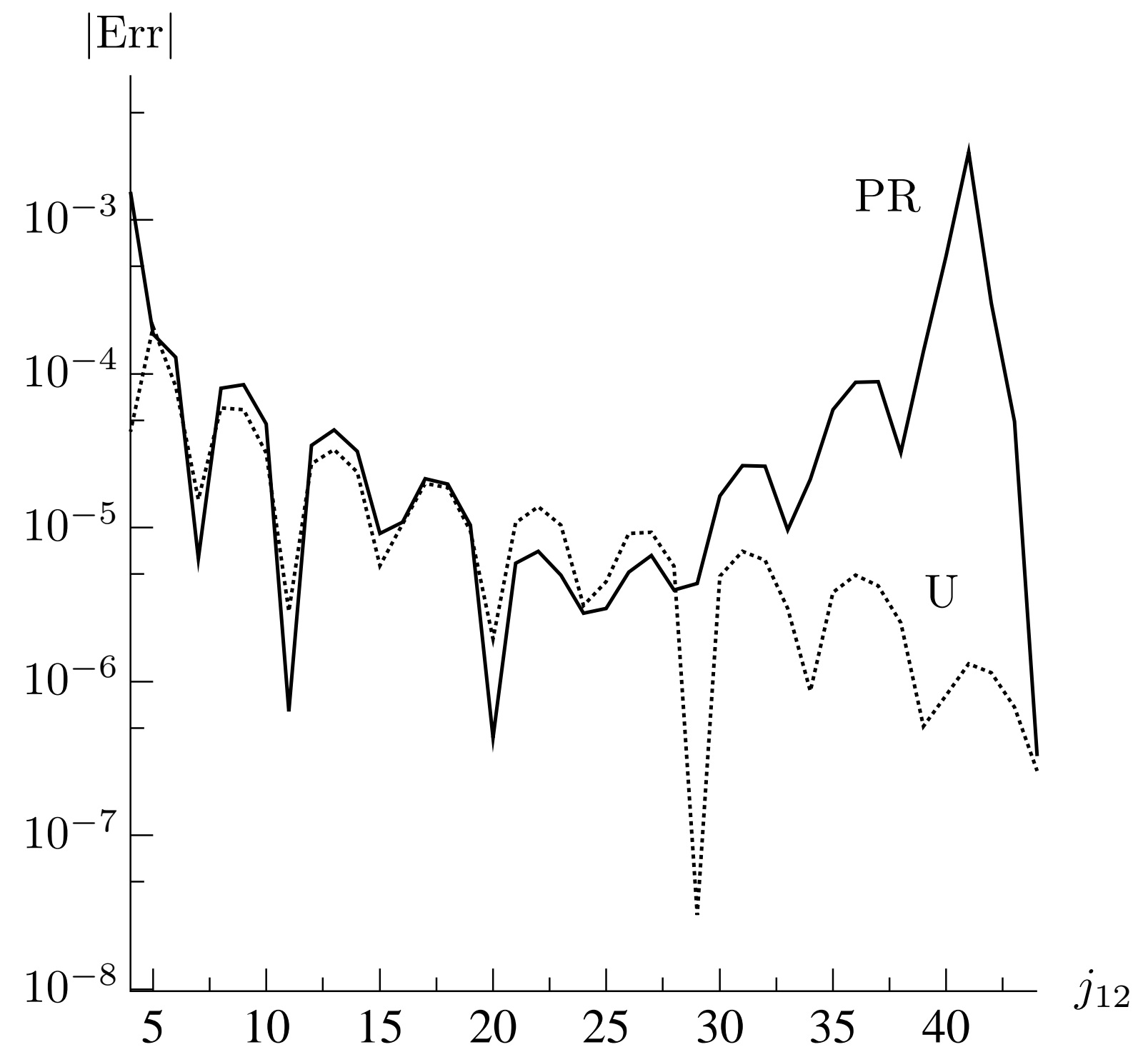



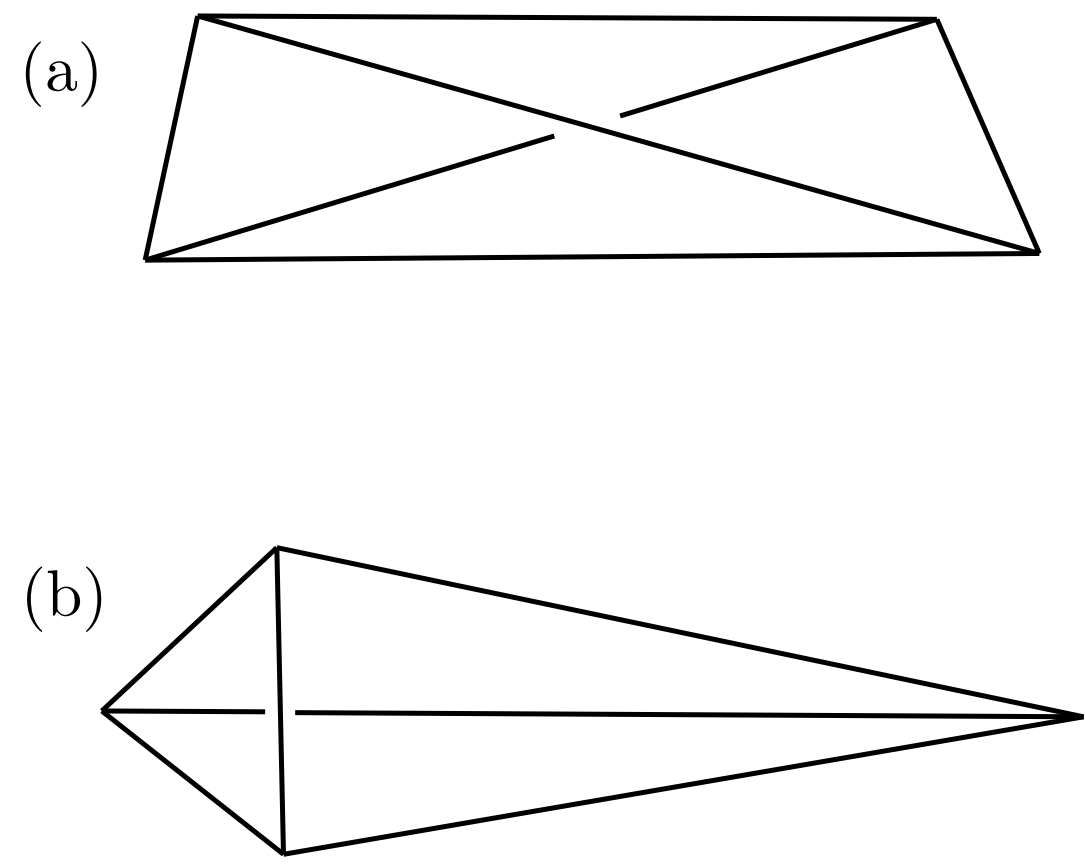


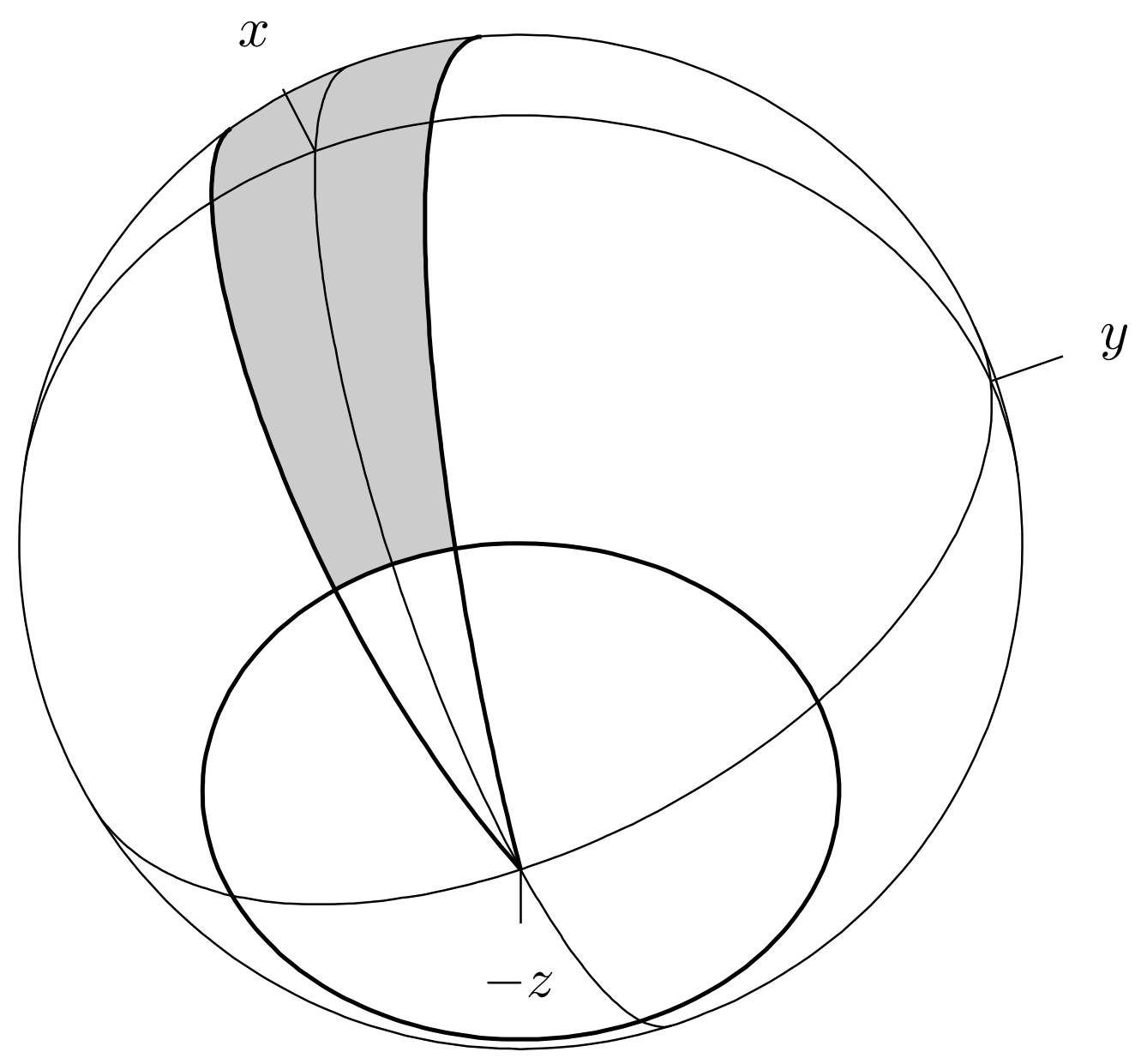

